PHYSICAL REVIEW D 89, 114003 (2014)

\title{
Production of charmed pseudoscalar mesons in antiproton-proton annihilation
}

\author{
J. Haidenbauer ${ }^{1}$ and G. Krein ${ }^{2}$ \\ ${ }^{1}$ Institute for Advanced Simulation and Jülich Center for Hadron Physics, \\ Forschungszentrum Jülich, D-52425 Jülich, Germany \\ ${ }^{2}$ Instituto de Física Teórica, Universidade Estadual Paulista, Rua Dr. Bento Teobaldo Ferraz, \\ 271 - Bloco II, 01140-070 São Paulo, São Paulo, Brazil
}

(Received 18 April 2014; published 3 June 2014)

\begin{abstract}
We study the production of charmed mesons $\left(D, D_{s}\right)$ in antiproton-proton $(\bar{p} p)$ annihilation close to the reaction thresholds. The elementary charm production process is described by baryon exchange and in the constituent quark model. Effects of the interactions in the initial and final states are taken into account rigorously. The calculations are performed in close analogy to our earlier study on $\bar{p} p \rightarrow \bar{K} K$ by connecting the processes via $\mathrm{SU}(4)$ flavor symmetry. Our predictions for the $D \bar{D}$ production cross section are in the order of $10^{-2}-10^{-1} \mu \mathrm{b}$. They turned out to be comparable to those obtained in other studies. The cross section for a $D_{s}^{+} D_{s}^{-}$pair is found to be of the same order of magnitude despite the fact that its production in $\bar{p} p$ scattering requires a two-step process.
\end{abstract}

DOI: $10.1103 /$ PhysRevD.89.114003

PACS numbers: 13.60.Le, 13.75.-n, 14.40.Lb, 25.43.+t

\section{INTRODUCTION}

Physics involving charmed particles is one of the main topics to be explored at the planned FAIR facility in Darmstadt $[1,2]$. In particular, the program proposed by the PANDA Collaboration encompasses a wide range of activities connected to this subject including high-accuracy spectroscopy of charmed hadrons and the investigation of their interactions with ordinary matter [3]. Presently very little is known about the interaction of charmed particles with conventional hadrons and/or nuclear matter built up predominantly from up and down quarks. Clearly, the rate at which charmed hadrons can be produced is a crucial factor for designing and performing secondary experiments with those particles. In particular, attaining a sufficient yield is a prerequisite for investigating issues like $c \bar{c}$ quarkonium dissociation [4] and the creation of new exotic nuclear bound states of $J / \psi$ and $\eta_{c}$ [5-9], charmed hypernuclei [10], and charmed D-mesic nuclei [11-13] that have been discussed in the literature over the last few years.

In this work we present predictions for the charmproduction reactions $\bar{p} p \rightarrow D \bar{D}$ and $\bar{p} p \rightarrow D_{s}^{+} D_{s}^{-}$close to their thresholds. The work builds on the Jülich mesonbaryon model for the reaction $\bar{p} p \rightarrow \bar{K} K$ [14-16]. The extension of the model from the strangeness to the charm sector follows a strategy similar to our recent work on the $D N$ and $\bar{D} N$ interactions [17-19], and on the reaction $\bar{p} p \rightarrow \bar{\Lambda}_{c}^{-} \Lambda_{c}^{+}$[20], namely, by imposing as a working hypothesis SU(4) symmetry constraints and improvements from quark-gluon dynamics at short distances $[21,22]$. The microscopic charm-production process is described by baryon exchange $\left(\Lambda_{c}, \Sigma_{c}\right)$, and the transition potentials are derived from the corresponding transitions in the strangeness-production channels $(\bar{K} K)$ using values of the involved coupling constants that are fixed from SU(4) symmetry. The reaction amplitudes themselves are evaluated in distorted-wave Born approximation (DWBA). This is done because we want to take into account rigorously the effects of the initial $(\bar{p} p)$ and also of the final-state interactions which are known to play an important role for energies near the production threshold [23-28].

As before in our study of the reaction $\bar{p} p \rightarrow \bar{\Lambda}_{c}^{-} \Lambda_{c}^{+}$[20], we investigate the effect of replacing the transition interaction based on meson-baryon dynamics by a charmproduction potential derived in the constituent quark model. This allows us to shed light on the model dependence of our results. Furthermore, we compare our predictions with the ones of other model calculations of the $\bar{p} p \rightarrow D \bar{D}$ reaction from the literature [29-34]. In some of those studies, a quark-gluon description based on a factorization hypothesis of hard and soft processes $[29,34]$ is employed, while in others a nonperturbative quark-gluon string model is used, based on secondary Regge pole exchanges including absorptive corrections $[30,32,33]$. Preliminary results (for $\bar{p} p \rightarrow D \bar{D}$ ) of our study were presented in Ref. [35].

The paper is organized as follows. In Sec. II we discuss the $\bar{N} N$ interaction used for the initial-state interaction (ISI). Because of the known sensitivity of the annhilation cross sections on the ISI, we examine its effect by considering various $\bar{N} N$ potentials where we make sure that all of them reproduce the total $\bar{p} p$ cross section in the relevant energy range and, in general, describe also data on integrated elastic and charge-exchange cross sections and even $\bar{p} p$ differential cross sections. Predictions for the reaction $\bar{p} p \rightarrow D \bar{D}$ that include effects of the $\bar{p} p$ ISI are presented in Sec. III. Transition potentials based on 
meson-baryon dynamics and derived in the quark model are considered.

To study also the influence of the final-state interaction (FSI), we extend a $\pi \pi-\bar{K} K$ interaction potential, developed by the Jülich group in the past [36,37], by adding to this coupled-channel model the $D \bar{D}$ and the $D_{s}^{+} D_{s}^{-}$ channels. This extension is described in detail in Sec. IV, and then the influence of the resulting FSI on the $\bar{p} p \rightarrow D \bar{D}$ cross section is examined. Finally, we provide predictions for the production of the charmed strange meson $D_{s}$ in the reaction $\bar{p} p \rightarrow D_{s}^{+} D_{s}^{-}$. In this case a two-step process is required, and, therefore, the pertinent cross section cannot be calculated in approaches that rely on the Born approximation in one way or the other. However, in a coupled-channel approach like ours, the transition amplitude from $\bar{p} p$ to $D_{s}^{+} D_{s}^{-}$is generated in a natural way. The paper ends with a summary. Technical aspects related to the derivation of the various interaction potentials are summarized in the Appendices.

\section{INTERACTION IN THE INITIAL $\bar{N} N$ SYSTEM}

For the $\bar{N} N$ interaction in the initial state, we take the same model that has been already employed in our recent study of the process $\bar{p} p \rightarrow \bar{\Lambda}_{c}^{-} \Lambda_{c}^{+}$[20]. It is based on the interaction originally developed for our investigation of $\bar{p} p \rightarrow \bar{\Lambda} \Lambda$ and consists of an elastic part which is deduced (via G-parity transform) from a simple, energy-independent one-boson-exchange $N N$ potential (OBEPF) [38] and a phenomenological annihilation part for which a spin-, isospin-, and energy-independent optical potential of Gaussian form is adopted:

$$
V_{o p t}^{\bar{N} N \rightarrow \bar{N} N}(r)=\left(U_{0}+i W_{0}\right) e^{-r^{2} / 2 r_{0}^{2}} .
$$

The parameters of the potential $\left(U_{0}, W_{0}, r_{0}\right)$ can be found in Ref. [20]. They were determined by a fit to $\bar{N} N$ data in the energy range relevant for the reaction $\bar{p} p \rightarrow \bar{\Lambda}_{c}^{-} \Lambda_{c}^{+}$, specifically to total cross sections [39-41] around $p_{\text {lab }}=10 \mathrm{GeV} / \mathrm{c}$, i.e., close to the $\bar{\Lambda}_{c}^{-} \Lambda_{c}^{+}$threshold, which is at $10.162 \mathrm{GeV} / \mathrm{c}$. A comparison of the model results with the data on total and integrated elastic and charge-exchange cross sections but also with differential $\bar{p} p$ cross sections $[40,42]$ around $10 \mathrm{GeV} / \mathrm{c}$ was presented in Ref. [20].

The thresholds for the reactions $\bar{p} p \rightarrow D \bar{D}$ and $\bar{p} p \rightarrow$ $D_{s}^{+} D_{s}^{-}$are at somewhat lower momenta, namely, at 6.442 and $7.255 \mathrm{GeV} / \mathrm{c}$, respectively. Therefore, we present here again $\bar{N} N$ results, but now we compare them with experiments over a momentum range that covers also the thresholds of those two reactions we study in the present work. Integrated cross sections for the considered $\bar{N} N$ interactions are summarized in Table I.

As already noted in Ref. [20], at the high energies where $D$ and $D_{s}$ production occurs, any $N N$ potential has to be considered as being purely phenomenological, and,
TABLE I. Total and integrated elastic $\bar{p} p$ cross sections and integrated charge-exchange ( $\bar{p} p \rightarrow \bar{n} n)$ cross sections in $m b$ for the four potentials considered in comparison to experimental values.

\begin{tabular}{ccccccc}
\hline \hline & $\begin{array}{c}p_{\text {lab }} \\
(\mathrm{GeV} / \mathrm{c})\end{array}$ & $\mathrm{A}$ & $\mathrm{A}^{\prime}$ & $\mathrm{B}$ & $\mathrm{C}$ & Experiment \\
\hline$\sigma_{\text {tot }}$ & 6.65 & 56.6 & 59.1 & 57.0 & 56.9 & $59.5 \pm 0.5[39]$ \\
& 7.30 & 56.0 & 58.5 & 56.3 & 56.3 & $58.3 \pm 1.3[43]$ \\
& 9.10 & 54.7 & 56.9 & 54.7 & 54.8 & $57.51 \pm 0.73[44]$ \\
& 10.0 & 54.2 & 56.4 & 54.1 & 54.3 & $54.7 \pm 0.60[39]$ \\
& 12.0 & 53.5 & 55.6 & 53.3 & 53.4 & $51.7 \pm 0.80[45]$ \\
$\sigma_{\text {el }}$ & 6.0 & 15.9 & 15.1 & 16.7 & 15.9 & $15.6 \pm 0.8[46]$ \\
& 7.2 & 15.2 & 14.3 & 15.8 & 15.2 & $13.79 \pm 1.0[40]$ \\
& 8.0 & 14.9 & 14.0 & 15.4 & 14.8 & $12.88 \pm 0.1[47]$ \\
& 8.9 & 14.6 & 13.6 & 15.0 & 14.5 & $13.89 \pm 0.35[40]$ \\
& 10.0 & 14.4 & 13.4 & 14.6 & 14.2 & $14.6 \pm 3.3[40]$ \\
& 12.0 & 14.0 & 13.0 & 14.1 & 13.8 & $11.59 \pm 0.41[40]$ \\
& & & & & & $11.34 \pm 0.6[45]$ \\
$\sigma_{\text {cex }}$ & 6.0 & 0.50 & 0.57 & 0.55 & 0.78 & $0.563 \pm 0.082[41]$ \\
& 7.0 & 0.38 & 0.45 & 0.42 & 0.64 & $0.373 \pm 0.054[41]$ \\
& 7.76 & 0.32 & 0.37 & 0.36 & 0.57 & $0.380 \pm 0.042[48]$ \\
& 9.0 & 0.24 & 0.29 & 0.28 & 0.47 & $0.284 \pm 0.041[41]$ \\
\hline \hline
\end{tabular}

therefore, one has to question whether fixing the elastic part of the $\bar{N} N$ potential via G parity by using such an $N N$ interaction that was fitted to low-energy $N N$ data is still meaningful. In addition, one knows from studies on $\bar{p} p \rightarrow$ $\bar{\Lambda} \Lambda$ that the magnitude of the cross sections depends very sensitively on the ISI [23,25-27]. Specifically, the absorptive character of the $\bar{N} N$ interaction leads to a strong reduction of the cross section as compared to results obtained in the Born approximation, i.e., based on the transition potential alone. Because of these reasons, in Ref. [20] several variants of the $\bar{N} N$ model were considered which differed in the treatment of the elastic part, with the intention to use them for illustrating the uncertainties in the predictions due to the used $\bar{p} p$ interaction. In all those $\bar{N} N$ interaction potentials (denoted by $\mathrm{A}, \mathrm{B}, \mathrm{C}$, and $\mathrm{D}$ in Ref. [20]), the longest ranged (and model-independent) part of the elastic $\bar{p} p$ interaction, namely, one-pion exchange, was kept, but the shorter ranged contributions, consisting of vector-meson and scalar-meson exchanges, were treated differently. In the present investigation, we do not consider model $\mathrm{D}$, which does not provide a realistic description of the $\bar{N} N$ data in the energy region relevant for $D$ and $D_{s}$ production. Instead we consider a new fit, called $A^{\prime}$, that includes only one-pion exchange for the elastic part (like $A$ ) but yields a better reproduction of the somewhat stronger falloff of the differential cross section exhibited by the data around $6.2 \mathrm{GeV} / \mathrm{c}$; see Fig. 1. In any case, in all scenarios a rather satisfying description of the $\bar{N} N$ data in the region 6-10 GeV/c is obtained; cf. Table I and Fig. 1. In particular, not only the slope but in some cases even the shoulder in the differential cross section is reproduced quantitatively by these interactions. 

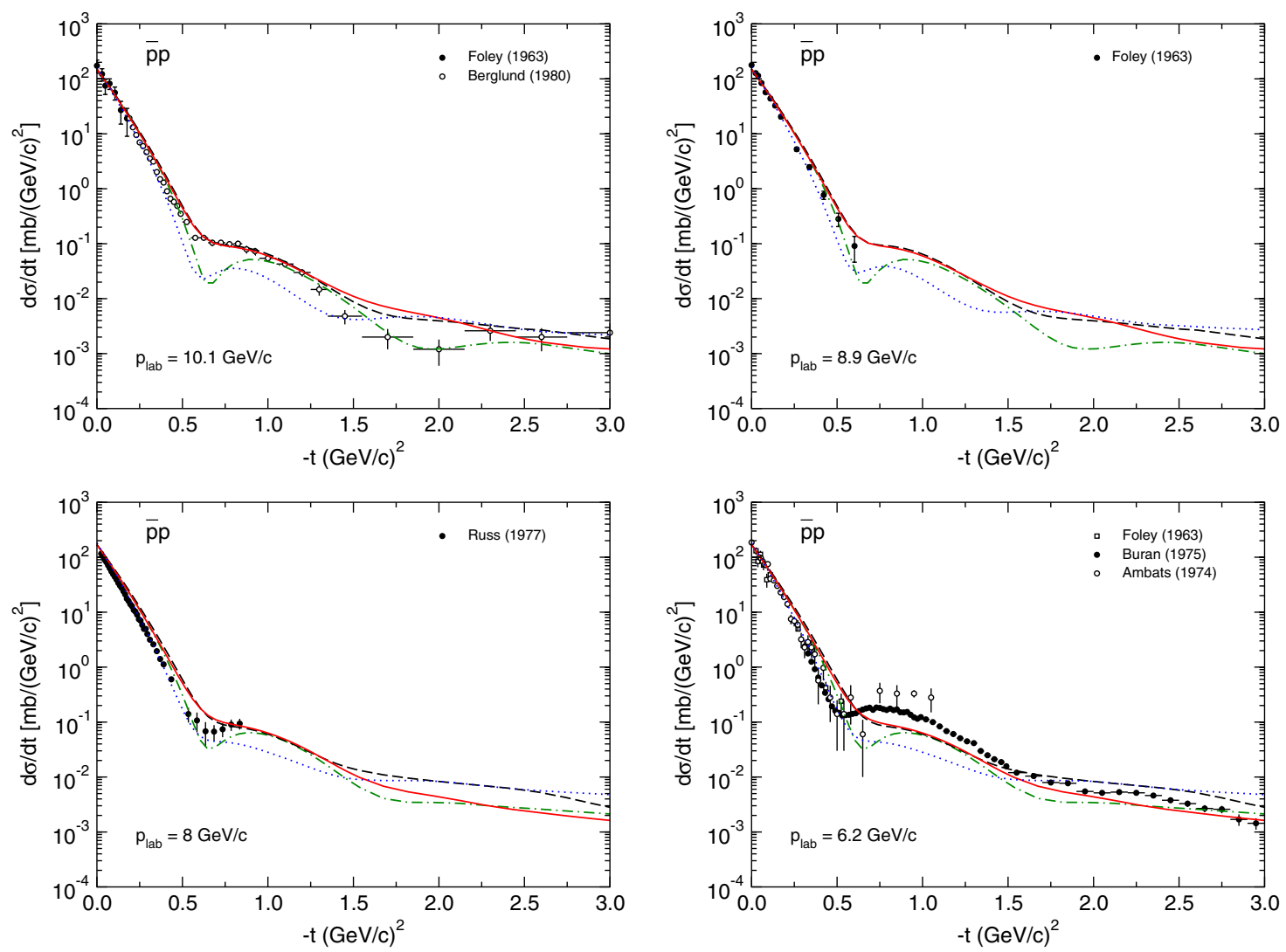

FIG. 1 (color online). Differential cross section for elastic $\bar{p} p$ scattering at $p_{\text {lab }}=6.2,8,8.9$, and $10.1 \mathrm{GeV} / \mathrm{c}$ as a function of $t$. The dashed-dotted curve corresponds to a calculation where only one-pion exchange is added to the optical potential (A). The dashed and solid curves are obtained by leaving out vector-meson exchanges (B) or by reducing the elastic part (except for the pion exchange) to $10 \%(\mathrm{C})$, respectively. The dotted line is an alternative fit, made to reproduce specifically the slope of the data at $6.2 \mathrm{GeV} / \mathrm{c}$, where likewise only pion exchange is added to the optical potential (A'). The experimental information is taken from Foley et al. [40], Berglund et al. [42], Russ et al. [47], Buran et al. [49], and Ambats et al. [46].

\section{REACTION $\bar{p} p \rightarrow D \bar{D}$}

\section{A. $\bar{p} p \rightarrow D \bar{D}$ based on baryon exchange}

Within meson-baryon dynamics, the transition from $\bar{p} p$ to $D \bar{D}$ is generated by the exchange of charmed baryons, in particular the $\Lambda_{c}$ and $\Sigma_{c}$ (in analogy to the exchange of $\Lambda$ and $\Sigma$ in case of the reaction $\bar{p} p \rightarrow \bar{K} K$ ); see Fig. 2 . Explicit expressions for the transition potentials can be found in Appendix A of Ref. [14]. They are of the generic form

$$
V^{\bar{N} N \rightarrow D \bar{D}}(t) \sim \sum_{Y=\Lambda_{c}^{+}, \Sigma_{c}, \Sigma_{c}^{*}} \frac{f_{Y N D}^{2} F_{Y N D}^{2}(t)}{\omega_{D}\left(\sqrt{s}-E_{N}-\omega_{D}-E_{Y}\right)},
$$

where $f_{Y N D}$ are coupling constants; $F_{Y N D}(t)$ are vertex form factors; and $E_{N}, \omega_{D}, E_{Y}$ are the energies of the nucleon, $D$-meson, and the exchanged baryon, respectively. Under the assumption of $S U(4)$ symmetry, the pertinent coupling constants are given by

$$
\begin{aligned}
f_{\Lambda_{c}^{+} N D} & =-\frac{1}{\sqrt{3}}(1+2 \alpha) f_{N N \pi} \approx-1.04 f_{N N \pi}, \\
f_{\Sigma_{c} N D} & =(1-2 \alpha) f_{N N \pi} \approx 0.2 f_{N N \pi},
\end{aligned}
$$

where we assumed for the $\mathrm{F} /(\mathrm{F}+\mathrm{D})$ ratio $\alpha \approx 0.4$. Thus, one expects that $\Lambda_{c}^{+}$exchange dominates the transition, while $\Sigma_{c}$ exchange should be suppressed. Specifically, the isospin decomposition

$$
\begin{aligned}
V^{\bar{p} p \rightarrow D^{0} \bar{D}^{0}} & =\frac{1}{2}\left(V_{I=0}^{\bar{N} N \rightarrow D \bar{D}}+V_{I=1}^{\bar{N} N \rightarrow D \bar{D}}\right), \\
V^{\bar{p} p \rightarrow D^{+} D^{-}} & =\frac{1}{2}\left(V_{I=0}^{\bar{N} N \rightarrow D \bar{D}}-V_{I=1}^{\bar{N} N \rightarrow D \bar{D}}\right),
\end{aligned}
$$

suggests that $V^{\bar{p} p \rightarrow D^{0} \bar{D}^{0}} \gg V^{\bar{p} p \rightarrow D^{+} D^{-}}$because the (dominant) contribution of the isoscalar $\Lambda_{c}^{+}$exchange drops out in the latter channel. Indeed, within the Born approximation, the cross sections predicted for $D^{0} \bar{D}^{0}$ are more than 2 orders of magnitude larger than those for $D^{+} D^{-}$; cf., the dotted lines Fig. 3. [The coupling constant $f_{N \Sigma^{*} K}$, and accordingly for $f_{N \Sigma_{c}^{*} D}$, is likewise very small [50] so that 


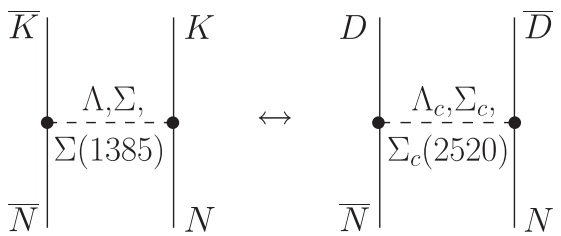

FIG. 2. Transition potential for $\bar{N} N \rightarrow D \bar{D}$ (right) and $\bar{N} N \rightarrow$ $\bar{K} K$ (left), respectively.

the contribution of $\Sigma^{*}\left(\Sigma_{c}^{*}\right)$ exchange turns out to be negligible.]

The vertex form factors adopted in Refs. [14,15] for the $\bar{N} N$ annihilation diagrams are not of the conventional monopole type but involve fourth powers of the cutoff mass $\Lambda$, of the exchanged baryon, and of the transferred momentum, see Eq. (2.15) in Ref. [14]. Such a more complicated parametrization was required in order to avoid unphysical singularities in the potential. We employ the same form here. In the actual calculation, a cutoff mass $\Lambda$ of $3.5 \mathrm{GeV}$ at the $Y N D$ vertices is used. This choice is motivated by the experience gained in our studies of $\bar{N} N \rightarrow$ $M M$ annihilation processes in the past and, specifically, in $\bar{N} N \rightarrow \bar{K} K$ where cutoff masses that are roughly $1 \mathrm{GeV}$
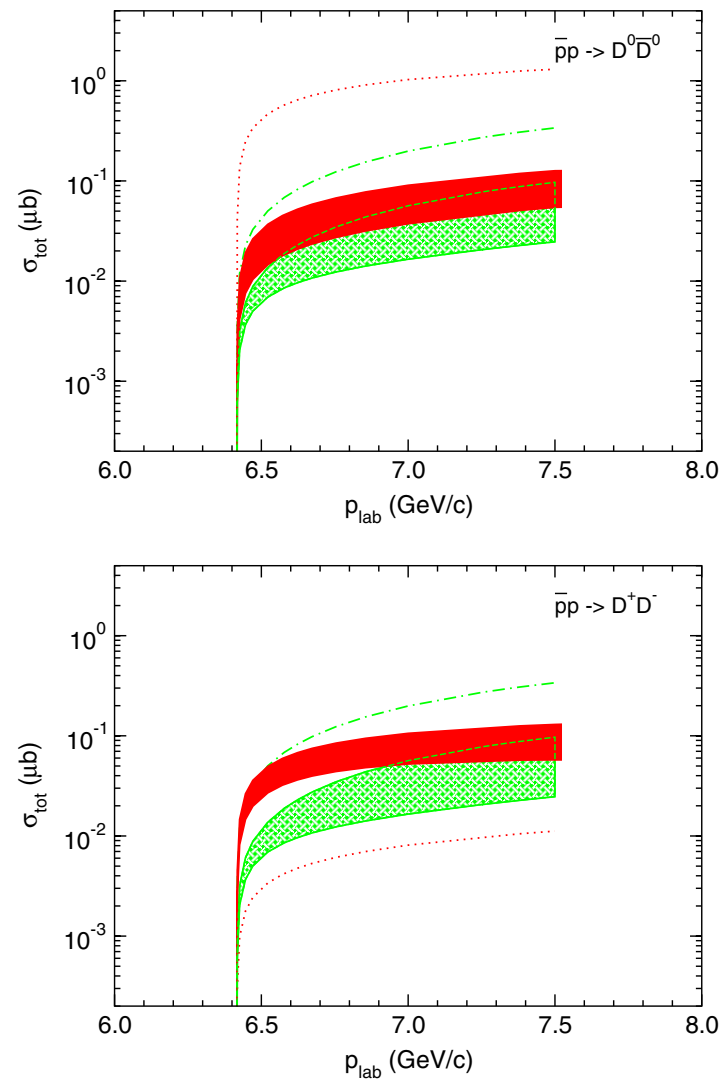

FIG. 3 (color online). Total reaction cross sections for $\bar{p} p \rightarrow$ $D \bar{D}$ as a function of $p_{\text {lab }}$, based on baryon exchange (shaded band) and the quark model (grid). Results obtained in the Born approximation are indicated by the dotted (baryon exchange) and dash-dotted (quark model) lines, respectively. larger than the masses of the exchanged baryons were found to be appropriate. We will come back to (and explore) the sensitivity of the results to variations of the cutoff mass below.

Let us now focus on the effects of the initial state interaction. Those effects are included by solving the formal coupled-channel equations

$$
\begin{aligned}
& T^{\bar{N} N, \bar{N} N}=V^{\bar{N} N, \bar{N} N}+V^{\bar{N} N, \bar{N} N} G^{\bar{N} N} T^{\bar{N} N, \bar{N} N}, \\
& T^{D \bar{D}, \bar{N} N}=V^{D \bar{D}, \bar{N} N}+V^{D \bar{D}, \bar{N} N} G^{\bar{N} N} T^{\bar{N} N, \bar{N} N},
\end{aligned}
$$

using the $\bar{N} N$ potential described in Sec. II. Of course, Eq. (6) implies that the $\bar{N} N \rightarrow D \bar{D}$ transition amplitude is effectively evaluated in a DWBA.

Results with the inclusion of ISI effects are presented as bands in Fig. 3 because we consider several variants of the $\bar{N} N$ potential as discussed in the previous section. It is obvious that the results change drastically once the ISI is included in the calculation. The cross sections for $D^{0} \bar{D}^{0}$ are strongly reduced, while at the same time those for $D^{+} D^{-}$ are enhanced. Indeed now both $D \bar{D}$ channels are produced at a comparable rate. In fact, the predicted cross section for $D^{+} D^{-}$appears to be even somewhat larger than the one for $D^{0} \bar{D}^{0}$.

Whereas the reduction in the $D^{0} \bar{D}^{0}$ case is in line with comparable effects observed in the previous studies of $\bar{N} N$ annihilation processes [23,25-27], as mentioned above, the enhancement seen for $D^{+} D^{-}$may be somewhat surprising, at least at first sight. However, it can be easily understood if one recalls that the $\Lambda_{c}^{+}$cannot contribute to the $\bar{p} p \rightarrow$ $D^{-} D^{+}$transition potential as discussed above. Only $\Sigma_{c}$ (and $\Sigma_{c}^{*}$ ) exchange contributes. But their coupling constants are very small according to SU(4) symmetry [cf. Eq. (3)], and the somewhat larger masses reduce the importance of $\Sigma_{c}$-exchange contributions further. This is the reason why the $\bar{p} p \rightarrow D^{+} D^{-}$cross section is strongly suppressed in the Born approximation. The consideration of the ISI via the employed DWBA approach (6) generates two-step transitions of the form $\bar{p} p \rightarrow \bar{n} n \rightarrow D^{+} D^{-}$. In this case $\Lambda_{c}^{+}$ exchange is no longer absent because it does contribute to the $\bar{n} n \rightarrow D^{+} D^{-}$transition potential, and, accordingly, those two-step transitions are enhanced in comparison to the Born approximation.

\section{B. $\bar{p} p \rightarrow D \bar{D}$ based on the quark model}

We consider a $\bar{p} p \rightarrow D \bar{D}$ transition potential derived in a constituent quark model where two light quark pairs $(\bar{u} u$ and $\bar{d} d$ ) are annihilated and a charmed quark pair ( $\bar{c} \mathrm{c})$ is created-see Fig. 4. We base our study on the model of Kohno and Weise [28] for the $\bar{p} p \rightarrow \bar{K} K$ reaction; we replace parameters corresponding to the $s$ quark and $K$ meson of that model by those of the $c$ quark and $D$ meson. The quark-model $\bar{N} N \rightarrow D \bar{D}$ transition potential $V_{Q}^{\bar{N} N \rightarrow D D}(t)$ can be written as 


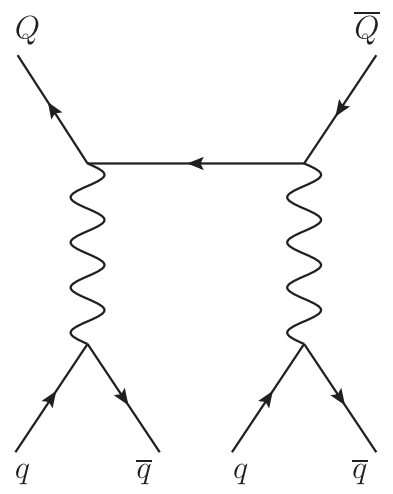

FIG. 4. Microscopic quark-model mechanism for the transition potential: annihilation of two pairs of light quarks, $q \bar{q}=u \bar{u}, d \bar{d}$, and creation of a pair of heavier quarks, $Q \bar{Q}=s \bar{s}, c \bar{c}$.

$$
V_{Q}^{\bar{N} N \rightarrow D \bar{D}}(t)=\chi_{\bar{N}}^{\dagger}\left[h_{1}(t) \boldsymbol{\sigma} \cdot \mathbf{p}+h_{2}(t) \boldsymbol{\sigma} \cdot \mathbf{p}^{\prime}\right] \chi_{N},
$$

where $\mathbf{p}$ and $\mathbf{p}^{\prime}$ are the $\bar{N} N$ and $D \bar{D}$ center-of-mass (c.m.) momenta, $\chi_{N}$ and $\chi_{\bar{N}}$ are the spin Pauli spinors of the nucleon and antinucleon, and $h_{1}(t)$ and $h_{2}(t)$ depend upon quark masses and hadron sizes and the effective strength of quark-pair annihilation and creation-their explicit expressions are given in Appendix B. A specific feature of the quark-model potential is that $V_{Q}^{\bar{p} p \rightarrow D^{0} \bar{D}^{0}}=-V_{Q}^{\bar{p} p \rightarrow D^{+} D^{-}}$(see Appendix B), so that there is no isospin $I=0$ transition. This is in contrast to the transitions induced by $\Lambda_{c}^{+}$and $\Sigma_{c}$ exchange, as discussed above.

Before presenting the results for $\bar{p} p \rightarrow D \bar{D}$, let us first examine the performance of the model in the reaction $\bar{p} p \rightarrow K^{-} K^{+}$for which there are experimental data available. We use standard quark-model values for quark masses and size parameters (they are given in Appendix B). And to facilitate a comparison with the results of Kohno and Weise, we use the same value for the effective coupling strength $\alpha_{A} / m_{G}^{2}$ as in their study of that reaction, namely, $\alpha_{A} / m_{G}^{2}=0.15 \mathrm{fm}^{2}$. The employed ISI is the same as for the $D \bar{D}$ case discussed above, but with parameters of the optical potential fitted to low-energy $\bar{N} N$ data (cf. OBEPF in Table IV of Ref. [51]). As visible from Fig. 5 (dashed line), the result is roughly in line with the available data, and it is also close to the original result of Kohno and Weise [28]. The differences are presumably due to the different ISI used by them and by us. Actually, with a slight reduction of the effective coupling strength $\left(\alpha_{A} / m_{G}^{2}=0.12 \mathrm{fm}^{2}\right)$, the bulk of the $K^{-} K^{+}$data can be quantitatively reproduced; see the solid curve in the figure. Thus, we will use this smaller coupling constant in the following calculations of charmed meson production to be on the safe side.

The quark model results for $\bar{p} p \rightarrow D \bar{D}$ are shown in Fig. 3. Clearly, because the transitions $D^{+} D^{-}$and $D^{0} \bar{D}^{0}$ are of the same magnitude, the corresponding cross sections calculated in Born approximation are the same. Moreover,

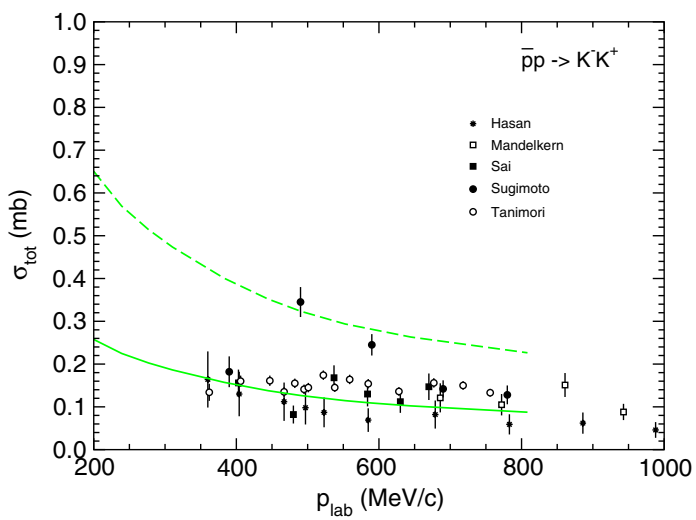

FIG. 5 (color online). Cross section for $\bar{p} p \rightarrow K^{-} K^{+}$scattering as a function of $p_{l a b}$. Results are based on the quark model. The curves correspond to different values for the effective coupling strength $\alpha_{A} / m_{G}^{2}-0.12 \mathrm{fm}^{2}$ (solid line) or $0.15 \mathrm{fm}^{2}$ (dashed line)-see the discussion in the text. Data are taken from Refs. [52-56].

for the same reason, the two-step transitions $\bar{p} p \rightarrow \bar{N} N \rightarrow$ $D^{+} D^{-}$and $\bar{p} p \rightarrow \bar{N} N \rightarrow D^{0} \bar{D}^{0}$ that make up the ISI provide equal reductions for both final states. Figure 3 also reveals that the quark model and baryon-exchange transitions yield comparable predictions, with those of the quark model being on average smaller by a factor roughly equal to 3 . In addition, the results show once more the fundamental role played by the ISI in the $\bar{p} p$ annihilation process, as the two transition mechanisms have very different isospin dependence and yet the final results are of comparable magnitude.

Predictions for the differential cross sections based on the baryon-exchange transition potential are presented in Fig. 6 at the excess energy $\epsilon=40 \mathrm{MeV}$ (corresponding to $p_{\text {lab }}=6.578 \mathrm{GeV} / \mathrm{c}$ ). We show the results for the different ISI separately so that one can see the variations induced by the individual $\bar{N} N$ potentials. The overall variation at this energy amounts to roughly a factor 2 . In all cases there is only a rather weak dependence of the $D^{0} \bar{D}^{0}$ and $D^{+} D^{-}$ cross sections on the scattering angle, which is a clear sign for the dominance of $s$-wave production. This is not surprising in view of the fact that the production mechanism is of rather short range. In this context it is instructive to recall the selection rules for the production of two pseudoscalar mesons [14]. Conservation of total angular momentum and parity implies that the lowest two partialwave amplitudes are given by the transitions ${ }^{3} P_{0} \rightarrow s$ and ${ }^{3} S_{1} \rightarrow p$, where the first symbol characterizes the $\bar{N} N$ partial wave in the standard spectral notation and the second specifies the angular momentum in the $D \bar{D}$ (or $\bar{K} K$ ) system. Dominance of the $s$ wave is therefore expected near the $D \bar{D}$ threshold. However, in the case of $\bar{p} p \rightarrow \bar{K} K$, one is actually close to the $\bar{p} p$ threshold so that the $\bar{N} N$ system is in the ${ }^{3} S_{1}$ partial wave and the $\bar{K} K$ system will be dominantly produced in a $p$ wave. Indeed, for that reaction, one observes a pronounced angular dependence of 

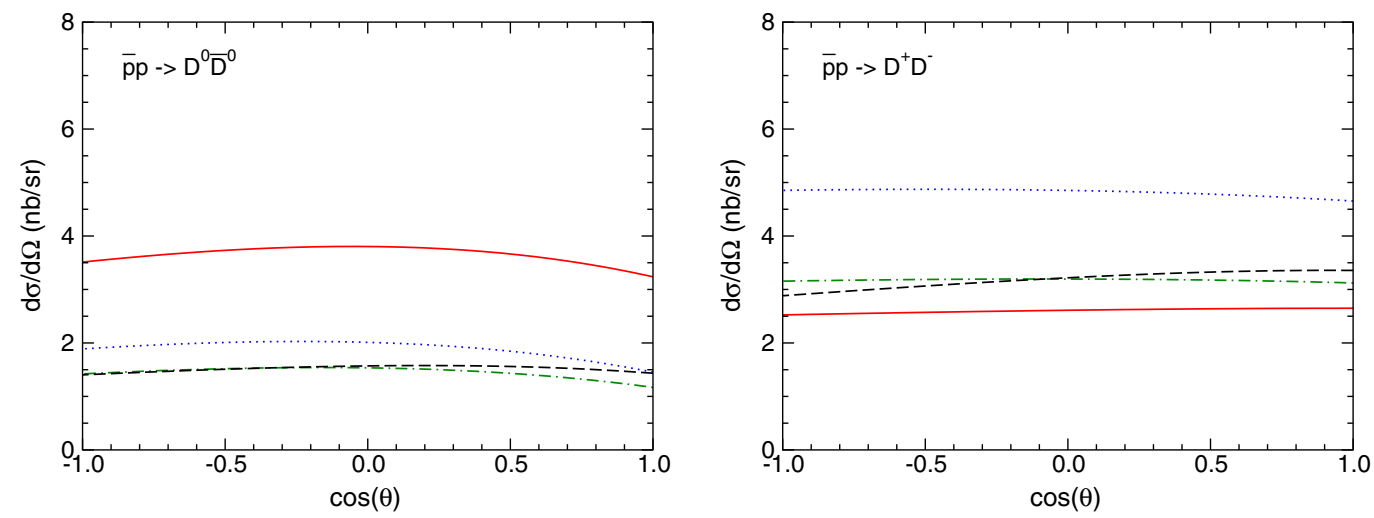

FIG. 6 (color online). Differential cross sections for $\bar{p} p \rightarrow D \bar{D}$ at $p_{\text {lab }}=6.578 \mathrm{GeV} / \mathrm{c}$ (excess energy $\epsilon=40 \mathrm{MeV}$ ). Results for different ISI are shown, namely for model A (dash-dotted curve), A' (dotted curve), B (dashed curve), and C (solid curve).

the differential cross section already at moderate energies, in the experimental data but also in model calculations [15].

The differential cross sections for $\bar{p} p \rightarrow D \bar{D}$ based on the constituent quark model exhibit a very similar behavior, and, therefore, we refrain from showing them.

Finally, let us mention that reducing the cutoff mass $\Lambda$ from 3.5 to $3 \mathrm{GeV}$ in the baryon-exchange transition potential reduces the cross section by roughly a factor 5 . Thus, the cutoff dependence appears to be somewhat stronger here than what we observed for $\bar{p} p \rightarrow \bar{\Lambda}_{c}^{-} \Lambda_{c}^{+}$ where the cross section dropped by a factor of around 3 for a comparable variation of the cutoff mass [20]. The variation of the cutoff mass simulates to some extent a possible SU(4) breaking in the $Y N D$ coupling constants because, like a direct variation of the coupling constants, it changes the strength of the potential in the relevant (physical) region of the momentum transfer $t$. Indeed, results for the $\Lambda_{c} N D$ and $\Sigma_{c} N D$ coupling constants from QCD sum rules [33] suggest a moderate breaking of SU(4) symmetry. Interestingly, the coupling constants for charmed baryons turned out to be somewhat larger than their strange counterparts, which, naively seen, would imply larger cross sections. In particular, the reported breaking of the SU(4) symmetry of $1.47_{-0.44}^{+0.58}$ in terms of the ratio of the $\Lambda_{c} N D$ to $\Lambda N K$ coupling constants [33] amounts to roughly a factor 5 on the level of the cross sections for the central value. Unfortunately, the theoretical uncertainty for the ratio is large so that, in principle, its value is even compatible with 1, i.e., with the $\mathrm{SU}(4)$ result. In any case, it is worthwhile to note that the variation in the cross sections deduced from the SU(4) breaking in the coupling constants is of very similar magnitude as the one suggested by our variation of the cutoff mass. In this context let us say that only a very small deviation from SU(4) symmetry, i.e., on the order of 1.05 in terms of the ratio of the $\Lambda_{c} N D$ to $\Lambda N K$ coupling constants, is obtained within the ${ }^{3} P_{0}$ constituent quark model [57].

The comparison between the results based on baryon exchange and on the quark model provides an alternative picture for the uncertainty in the $D \bar{D}$ production cross section, independent from the issue of SU(4) symmetry breaking. Also here we see variations in the order of a factor $3-5$, as mentioned above.

\section{Comparison with other results}

In the literature one can find several other studies of the reaction $\bar{p} p \rightarrow D \bar{D}$. The most recent publication is by Goritschnig et al. [34], who employ a quark-gluon description based on a factorization hypothesis of hard and soft processes. This work supersedes an earlier study by that group within a quark-diquark picture, where already concrete predictions for the $D^{+} D^{-}$production cross section were given [29]. In the study by Kaidalov and Volkovitsky [30], a nonperturbative quark-gluon string model was used, based on secondary Regge pole exchanges including absorptive corrections. On the same lines, there is the more recent publication by Titov and Kämpfer [32]. Finally, in the work by Khodjamirian et al. [33], the quark-gluon string model of Ref. [30] was revisited, but now strong coupling constants calculated from QCD lightcone sum rules are employed.

Interestingly, and in contrast to studies of the reaction $\bar{p} p \rightarrow \bar{\Lambda}_{c}^{-} \Lambda_{c}^{+}$[20], the majority of the calculations for $\bar{p} p \rightarrow D \bar{D}$ predict cross sections that are pretty much of comparable magnitude, at least on a qualitative level. This is to some extent surprising because, as far as we can see, none of the other studies take into account effects of the ISI, which strongly influences the magnitude of our results. Of course, one could argue that such effects are included effectively in the coupling constants or the diquark form factor, say, employed in those other studies. Anyway, the results presented in Ref. [32] as well as those in Ref. [33] exhibit a strong suppression of the $D^{+} D^{-}$cross section as compared to $D^{0} \bar{D}^{0}$-which, as we argued above, is definitely a consequence of the Born approximation together with $Y N D$ coupling constants that fulfill (approximate) SU(4) symmetry. 
On the quantitative level, we see that the $D^{0} \bar{D}^{0}$ cross section of Kaidalov [30] lies within the band of our results as provided in Fig. 3, and the same is true for the $D^{+} D^{-}$ cross section of Ref. [29] (in the energy region considered by us). The $D^{0} \bar{D}^{0}$ predictions in Ref. [33] are also comparable to ours, but their $D^{+} D^{-}$cross sections are down by 2 orders of magnitude. The $D \bar{D}$ cross section of Kerbikov [31] and the corrected $D^{0} \bar{D}^{0}$ cross section by Goritschnig et al. [34] (cf., the erratum) are about 1 order of magnitude smaller. In Ref. [32] only differential cross sections are given. Because of that we calculated $d \sigma / d t$ at the excess energy $\epsilon=0.5 \mathrm{GeV}$ and $t_{\max }-t=0.2 \mathrm{GeV}^{2}$ in order to facilitate a comparison. Our results for $\bar{p} p \rightarrow$ $D^{+} D^{-}$and $\bar{p} p \rightarrow D^{0} \bar{D}^{0}$ are $(0.8-1.8) \times 10^{-2}$ and $(0.6-1.3) \times 10^{-2} \mu b / \mathrm{GeV}^{2}$, respectively, which should be compared to $\approx 1.5 \times 10^{-2}$ and $\approx 50 \times 10^{-2} \mu b / \mathrm{GeV}^{2}$ by Titov and Kämpfer, estimated from their figures.

\section{EFFECTS OF THE FINAL $D \bar{D}$ INTERACTION AND THE REACTION $\bar{p} p \rightarrow D_{s}^{+} D_{s}^{-}$}

In the study of the reaction $\bar{p} p \rightarrow \bar{K} K$ by the Jülich group [16], the interaction in the $\bar{K} K$ channel was ignored. Indeed, since the mass of the kaon is significantly smaller than the one of the proton, already at the $\bar{p} p$ threshold the relative momentum in the produced $\bar{K} K$ system is fairly large, and, therefore, one can expect that FSI effects are small in this case. Moreover, as pointed out in Sec. III B, the $\bar{K} K$ pair is produced primarily in a $p$ wave near the $\bar{p} p$ threshold because of the section rules. Obviously, for $\bar{p} p \rightarrow$ $D \bar{D}$ these arguments no longer hold. Thus, in the following we want to investigate, at least qualitatively, the effect due to a FSI in the $D \bar{D}$ system, and we do this by adapting and extending a $\pi \pi-\bar{K} K$ (coupled channels) model developed by the Jülich group some time ago [36,37].

In the extension of the model, we include not only the $D \bar{D}$ channel but also the $D_{s}^{+} D_{s}^{-}$system. The mass of the charmed strange meson $D_{s}$ is with $1969 \mathrm{MeV}$, only about $100 \mathrm{MeV}$ larger than the one of the $D$ meson. Thus, the thresholds of those two channels are relatively close to each other, i.e., much closer than those of $D \bar{D}$ and $\bar{K} K$, say, which could be of relevance for the $D \bar{D}$ FSI effects. In addition, and more interestingly, taking into account the $D_{s}^{+} D_{s}^{-}$system enables us to provide also predictions for the reaction $\bar{p} p \rightarrow D_{s}^{+} D_{s}^{-}$because then annihilation into this channel becomes possible too, e.g., via the two-step process $\bar{p} p \rightarrow D \bar{D} \rightarrow D_{s}^{+} D_{s}^{-}$. The direct $\bar{p} p \rightarrow D_{s}^{+} D_{s}^{-}$ transition requires the annihilation of three (up or down) quark-antiquark pairs and a creation of two $(s$ and $c$ ) quarkantiquark pairs and is, therefore, suppressed by the OkuboZweig-Iizuka (OZI) rule.

The interactions in the $D \bar{D}$ and $D_{s}^{+} D_{s}^{-}$systems are constructed along the lines of the Jülich meson exchange model for the $\pi \pi$ interaction for which the evaluation has been discussed in detail in Refs. [36,37]. The present interaction is based on the version described in the latter
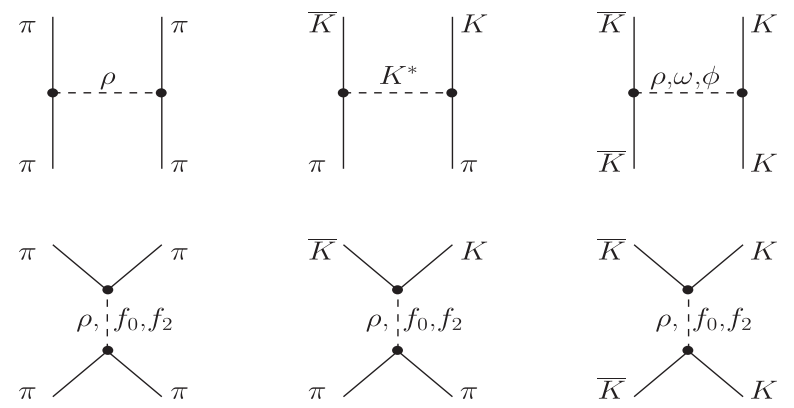

FIG. 7. Diagrams included in the Jülich $\pi \pi-\bar{K} K$ potential [37].

reference. The potentials for $\pi \pi \rightarrow \pi \pi, \pi \pi \rightarrow K \bar{K}$, and $K \bar{K} \rightarrow K \bar{K}$ are generated from the diagrams shown in Fig. 7. The figure contains only $s$ - and $t$-channel diagrams; $u$-channel processes corresponding to the considered $t$-channel processes are also included whenever they contribute. The scalar-isoscalar particle denoted by $\epsilon$ in Fig. 7 effectively includes the singlet and the octet member of the scalar nonet. The effects of $t$-channel $f_{2}(1270)$ and $\epsilon$ exchange were found to be negligible [37] and are, therefore, not included in the model.

The coupling constant $g_{\rho \pi \pi}$, required for $t$ - and $u$-channel exchange diagrams, is determined from the decay widths of the $\rho$. Most of the other coupling constants are determined from $\mathrm{SU}(3)$ symmetry relations, and standard assumptions about the octet/singlet mixing angles, as demonstrated in Ref. [36].

The scattering amplitudes are obtained by iterating these potentials by using a coupled channel scattering equation, formally given by

$$
T^{i, j}=V^{i, j}+\sum_{l} V^{i, l} G^{l} T^{l, j},
$$

with $i, j, l=\pi \pi, \pi \eta, K \bar{K}$.

This interaction yields a good description of the $\pi \pi$ phase shifts up to partial waves with total angular momentum $J=2$ and for energies up to $\sqrt{s} \approx 1.4 \mathrm{GeV}$, as can be seen in Ref. [37]. Furthermore, as a special feature, the $f_{0}(980)$ meson results as a dynamically generated state, namely, as a quasibound $\bar{K} K$ state. Also the $a_{0}(980)$ is found to be dynamically generated in the corresponding $\pi \eta-\bar{K} K$ system.

The additional diagrams that arise for the direct $D \bar{D}$ and $D_{s}^{+} D_{s}^{-}$potentials and for the transitions from $\pi \pi$ and/or $\bar{K} K$ to those channels are displayed in Fig. 8. In this extension we are again guided by SU(4) symmetry. Thus, we include $t$-channel exchanges of those vector mesons which are from the same SU(4) multiplet as those included in the original Jülich model, and, moreover, we assume that all coupling constants at the additional three-meson vertices are given by $\mathrm{SU}(4)$ relations. The latter are summarized in Appendix C. As can be seen in Fig. 7, the original Jülich 

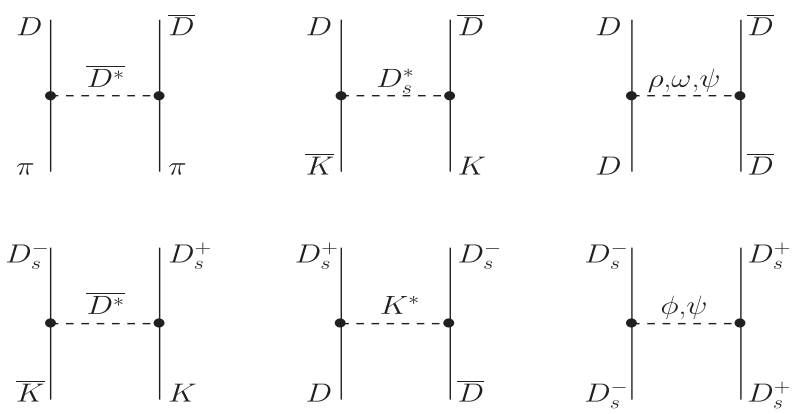

FIG. 8. Additional diagrams that contribute to the potential when the $D \bar{D}$ and $D_{s}^{-} D_{s}^{+}$channels are included.

model includes also $s$-channel (resonance) diagrams, specifically, $\pi \pi / K \bar{K} \rightarrow \epsilon, \rho, f_{2} \rightarrow \pi \pi / K \bar{K}$, which enable a unified description of all partial waves [37]. However, those resonances lie far below the thresholds of the $D \bar{D}$ and $D_{s}^{+} D_{s}^{-}$channels. Therefore, they have very little influence on the results for $D \bar{D}$ and $D_{s}^{+} D_{s}^{-}$scattering as we verified in test calculations where we assumed that the bare coupling constants of those resonances to $D \bar{D}$ are the same as those for $\bar{K} K$. Thus, in the present extension of the model [37] to the charm sector, we set their couplings to the $D \bar{D}$ system to zero.

Since the $D \bar{D}$ interaction was considered before and, specifically, in a meson-exchange approach [58], we display here also some prediction of the present model. Cross sections for $D \bar{D}$ scattering in the isospin $I=0$ and $I=1$ states can be found in Fig. 9. The main difference in the dynamics between our model and the one in Ref. [58] is that the latter includes also the exchange of scalar mesons. As mentioned above, $t$-channel exchange of a scalar meson has been considered in the original Jülich $\pi \pi-\bar{K} K$ potential [37] but was found to be negligible. Because of that we neglected contributions from scalar meson also in our extension to the charm sector.

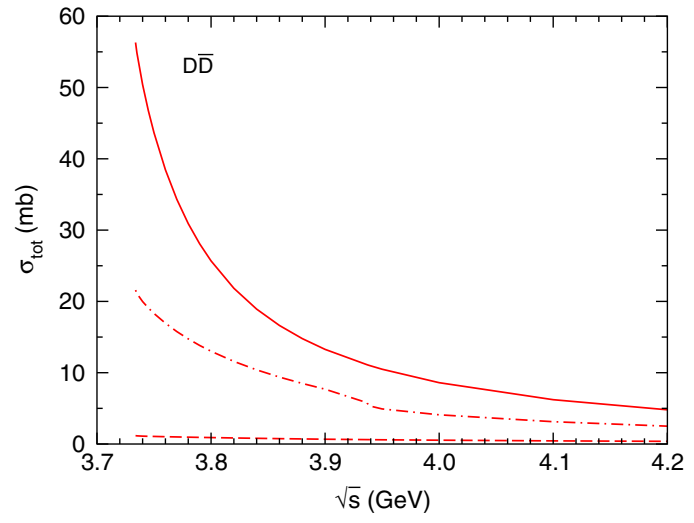

FIG. 9 (color online). Cross section for $D \bar{D}$ scattering in the $s$ wave as a function of $\sqrt{s}$. The solid line is the results for the isospin $I=0$ channel and the dashed line for the $I=1$ channel. The dashed-dotted curve indicates the changes in the $I=0$ case when the coupling to $\bar{D}_{s} D_{s}$ is included in the model.
In any case, we want to emphasize that one should not take the quantitative results too literally. It is obvious that without any constraints from experiments such model calculation are necessarily afflicted with sizeable uncertainties as is reflected in the results presented in Ref. [58]. The difference in the $\bar{D} D$ cross sections induced by the coupling to the $D_{s}^{+} D_{s}^{-}$system shown in Fig. 9 may serve as further illustration with regard to that. But the essential point for our purpose here is that the $D \bar{D}$ and $D_{s}^{+} D_{s}^{-}$ interactions incorporate all essential features one expects from a realistic FSI. Specifically the amplitudes are generated by solving a scattering equation; i.e., they fulfill unitarity requirements, and they include effects from the presence of open channels such as $\pi \pi$ and $\bar{K} K$.

The uncertainties of our predictions for the reactions $\bar{p} p \rightarrow D \bar{D}$ and $\bar{p} p \rightarrow D_{s}^{+} D_{s}^{-}$induced by the treatment of the $D \bar{D}$ and $D_{s}^{+} D_{s}^{-}$interactions are best estimated by simply switching off the corresponding FSI effects, which will be discussed below. Note that such a radical approach supersedes variations coming from a possible SU(4) breaking in the coupling constants involved in the $D \bar{D}$ and $D_{s}^{+} D_{s}^{-}$interactions, discussed in Appendix C.

Results for the reaction $\bar{p} p \rightarrow D \bar{D}$ are displayed in Fig. 10, where now only cross sections based on the $\bar{N} N$ interaction $A$ are presented so that one can distinguish the various FSI effects more clearly. For the other variants of the $\bar{N} N$ interaction, the effects are very similar. The dotted and dashed lines are again the results obtained in the Born approximation and by taking into account only the initial $\bar{N} N$ interaction, respectively. The inclusion of an interaction in the $D \bar{D}$ system (solid lines) yields a noticeable change in the energy dependence of the $D^{+} D^{-}$cross section and an enhancement in the case of the $D^{0} \bar{D}^{0}$ channel. The $D \bar{D}$ interaction is only strong in the $I=0$ channel, cf. Fig. 9, and, therefore, the inclusion of FSI effects modifies primarily the corresponding $I=0 \bar{N} N \rightarrow$ $D \bar{D}$ transition amplitude. Since the $D^{+} D^{-}$and $D^{0} \bar{D}^{0}$ production amplitudes are given by the coherent sum and difference of the $I=0$ and $I=1$ amplitudes [analogous to Eq. (4)], respectively, the $I=0$ amplitude interferes differently with the one for $I=1$ for the two particle channels, and, accordingly, the FSI effects are different.

Anyway, overall one can say that the changes are moderate, specifically if one recalls the variations due the ISI. The results do not change very much anymore when, finally, also the coupling to the $D_{s}^{+} D_{s}^{-}$channel is introduced (into the $D \bar{D}$ FSI) — see the dash-dotted linesthough there is a visible appearance of threshold effects from the opening of the $D_{s}^{+} D_{s}^{-}$channel in the $D^{+} D^{-}$as well as in the $D^{0} \bar{D}^{0}$ cross sections. Note that the coupling to the $D_{s}^{+} D_{s}^{-}$channel has a sizeable influence on the $D \bar{D}$ scattering cross section, as said before, see the dasheddotted curve in Fig. 9.

Our predictions for the reaction $\bar{p} p \rightarrow D_{s}^{+} D_{s}^{-}$can be found in Fig. 11, where we use the same scale as in the 

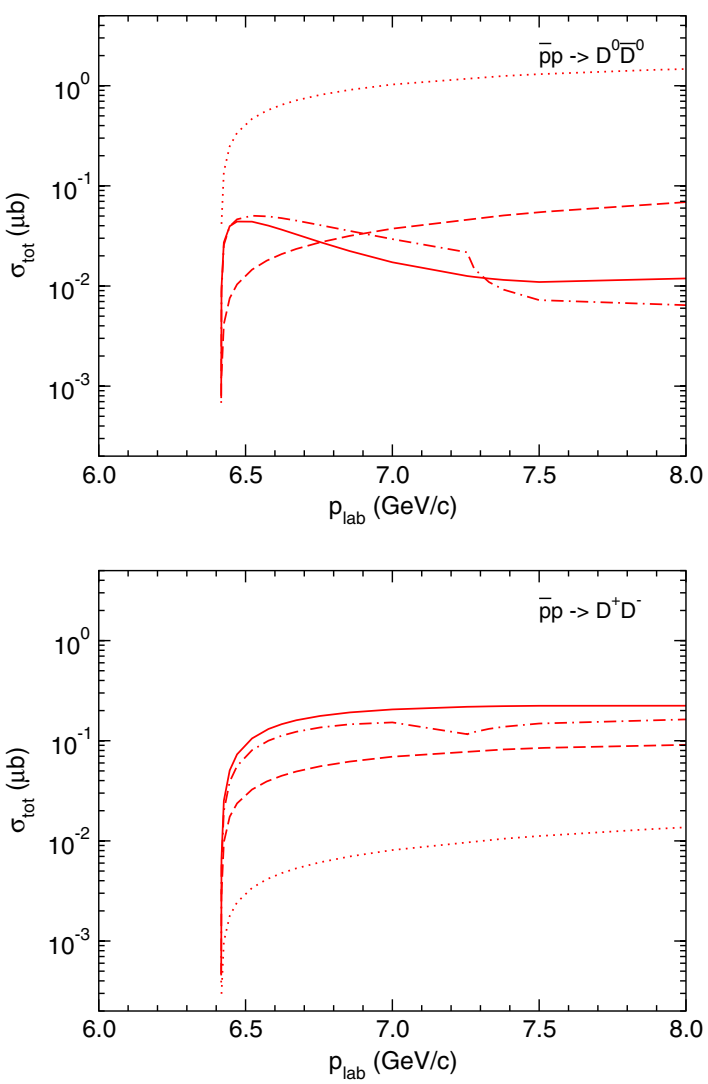

FIG. 10 (color online). Total reaction cross sections for $\bar{p} p \rightarrow$ $D \bar{D}$ as a function of $p_{\text {lab }}$. Effects of the final state interaction. The dashed lines are results with the $\bar{N} N$ model A as ISI, but without FSI. Inclusion of the $D \bar{D}$ FSI yields the solid curves. Including in addition the coupling of $D \bar{D}$ to $D_{s}^{+} D_{s}^{-}$leads to the dashed-dotted lines. Results obtained in the Born approximation are indicated by the dotted lines.

figures with the $\bar{p} p \rightarrow D \bar{D}$ results in order to facilitate a comparison. Thus, one can see easily that the cross sections for the two reactions are of comparable magnitude, even though a two-step process is required in the former. We should mention that this is not unusual. In a calculation of

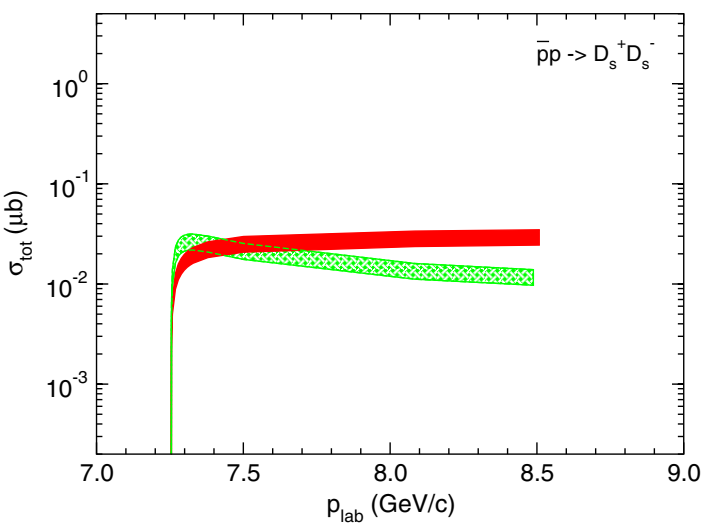

FIG. 11 (color online). Total reaction cross sections for $\bar{p} p \rightarrow$ $D_{s}^{+} D_{s}^{-}$as a function of $p_{\text {lab }}$, based on baryon exchange (shaded band) and the quark model (grid).
$\bar{\Sigma} \Sigma$ production, carried out in a similar framework by our group many years ago [59], it was found that the cross sections for $\bar{p} p \rightarrow \overline{\Sigma^{+}} \Sigma^{+}$and $\bar{p} p \rightarrow \overline{\Sigma^{-}} \Sigma^{-}$were of comparable magnitude. Also here the latter reaction requires (at least) a two-step process. Indeed, in that case an experiment performed several years later [60] confirmed that the $\overline{\Sigma^{-}} \Sigma^{-}$ production cross section is not suppressed at all.

With inclusion of the FSI, the amplitudes for $\bar{p} p \rightarrow D \bar{D}$ and $\bar{p} p \rightarrow D_{s}^{+} D_{s}^{-}$are given by

$$
T^{D \bar{D}, \bar{N} N}=\left(T^{D \bar{D}, D \bar{D}} G^{D \bar{D}}+1\right) V^{D \bar{D}, \bar{N} N}\left(1+G^{\bar{N} N} T^{\bar{N} N, \bar{N} N}\right)
$$

$$
\begin{aligned}
& T^{D_{s}^{+} D_{s}^{-}, \bar{N} N} \\
& \quad=T^{D_{s}^{+} D_{s}^{-}, D \bar{D}} G^{D \bar{D}} V^{D \bar{D}, \bar{N} N}\left(1+G^{\bar{N} N} T^{\bar{N} N, \bar{N} N}\right) \\
& \quad+T^{D_{s}^{+} D_{s}^{-}, \bar{K} K} G^{\bar{K} K} V^{\bar{K} K, \bar{N} N}\left(1+G^{\bar{N} N} T^{\bar{N} N, \bar{N} N}\right) .
\end{aligned}
$$

The coupled-channel formalism employed in our calculation implies that contributions from the two-step processes $\bar{p} p \rightarrow D \bar{D} \rightarrow D_{s}^{+} D_{s}^{-}$and $\bar{p} p \rightarrow \bar{K} K \rightarrow D_{s}^{+} D_{s}^{-}$are included (though it turned out that the latter one is negligibly small). In principle, there are many other twostep processes that lead likewise to a final $D_{s}^{+} D_{s}^{-}$state. Two examples are indicated by the diagrams on the right-hand side of Fig. 12. These are ignored in the present study but, of course, could affect the cross section. Nevertheless, we expect that the coupling to the $D^{*} \bar{D}^{*}$ channel should not change the $\left(D \bar{D}\right.$ and $\left.D_{s}^{+} D_{s}^{-}\right)$cross sections too dramatically, at least for energies below the $D^{*} \bar{D}^{*}$ threshold, based on what we saw in case of the $D \bar{D}$ results and the coupling to $D_{s}^{+} D_{s}^{-}$discussed above. There is another channel with open charm, namely, $D^{*} \bar{D}-D \bar{D}^{*}$, for which the threshold is between the ones for $D \bar{D}$ and $D_{s}^{+} D_{s}^{-}$. Fortunately, it contributes to different parity and total-angular-momentum states so that there is no coupling to the $D \bar{D}$ and $D_{s}^{+} D_{s}^{-}$ systems. The thresholds of other possible intermediate states that lead to a final $D_{s}^{+} D_{s}^{-}$system, like $\bar{p} p \rightarrow \bar{\Lambda} \Lambda \rightarrow$ $D_{s}^{+} D_{s}^{-}$, depicted also in Fig. 12 , or $\bar{p} p \rightarrow \bar{\Lambda}_{c}^{-} \Lambda_{c}^{+} \rightarrow D_{s}^{+} D_{s}^{-}$, say, are all far away from those of $D \bar{D}$ and $D_{s}^{+} D_{s}^{-}$, and, therefore, the corresponding two-step processes should be less important.

In any case, it is clear that the predicted $D_{s}^{+} D_{s}^{-}$cross section is afflicted with larger uncertainties than the one for
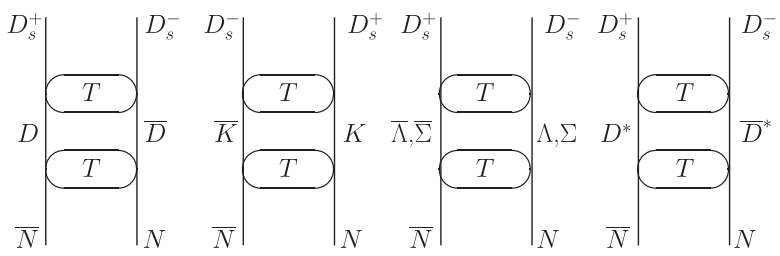

FIG. 12. Two-step processes that contribute to the reaction $\bar{N} N \rightarrow D_{s}^{+} D_{s}^{-} . T$ stands for the corresponding transition amplitudes. The two mechanisms on the left side are included in the present study. 
$D \bar{D}$. Still, we believe that the largest uncertainties come from the form factors in the transition potentials and from the $D_{s}^{+} D_{s}^{-}$interaction itself. Thus, as before in the $D \bar{D}$ case, we reduced the cutoff mass in the vertex form factor of the $\bar{p} p \rightarrow D \bar{D}$ transition potential from 3.5 to $3 \mathrm{GeV}$, and we also switched off the direct $D_{s}^{+} D_{s}^{-}$interaction in order to see its effect on the cross section. Both scenarios led to a reduction of the $D_{s}^{+} D_{s}^{-}$yield by a factor of around 4-5.

Finally, with the aim to shed light on the uncertainties from a different perspective, we derived also the mesonmeson $\bar{K} K \rightarrow D_{s}^{+} D_{s}^{-}$and $D \bar{D} \rightarrow D_{s}^{+} D_{s}^{-}$transition amplitudes from the quark-pair annihilation-creation processes $\bar{s} s \rightarrow \bar{c} c$ and $\bar{u} u \rightarrow \bar{s} s$, respectively; their explicit expressions are summarized in Appendix B. In Fig. 13 we present exemplary results for the cross section in the transition reaction $D \bar{D} \rightarrow D_{s}^{+} D_{s}^{-}$. One sees that the prediction obtained from the quark model is on average a factor of 4 smaller than the one from the meson-exchange model.

A direct calculation of $\bar{p} p \rightarrow D_{s}^{+} D_{s}^{-}$based on quarkmodel transition potentials yields a vanishing cross section. This is so because the transition $\bar{p} p \rightarrow D \bar{D}$ can only occur for isospin $I=1$ in the quark model, as said above, while the transition $D \bar{D} \rightarrow D_{s}^{+} D_{s}^{-}$can take place only in $I=0$ since $D_{s}^{+} D_{s}^{-}$is an $I=0$ system. Therefore, in the application to the $\bar{p} p \rightarrow D_{s}^{+} D_{s}^{-}$, we use (somewhat inconsistently) baryon-exchange amplitudes for $\bar{p} p \rightarrow D \bar{D}$ in order to estimate the effect of the quark model meson-meson transitions on the $\bar{p} p \rightarrow D_{s}^{+} D_{s}^{-}$reaction. Corresponding results are included in Fig. 11. Clearly, both the quark and the meson-exchange models yield predictions of comparable magnitude. This might be somewhat surprising in view of the cross sections shown in Fig. 13. However, one has to keep in mind that the latter is determined by the on-shell $D \bar{D} \rightarrow D_{s}^{+} D_{s}^{-} T$ matrix, while the $\bar{p} p \rightarrow D_{s}^{+} D_{s}^{-}$ amplitudes involve this amplitude off shell—see Eq. (10) and here the ones based on the quark and the mesonexchange models are obviously of similar magnitude.

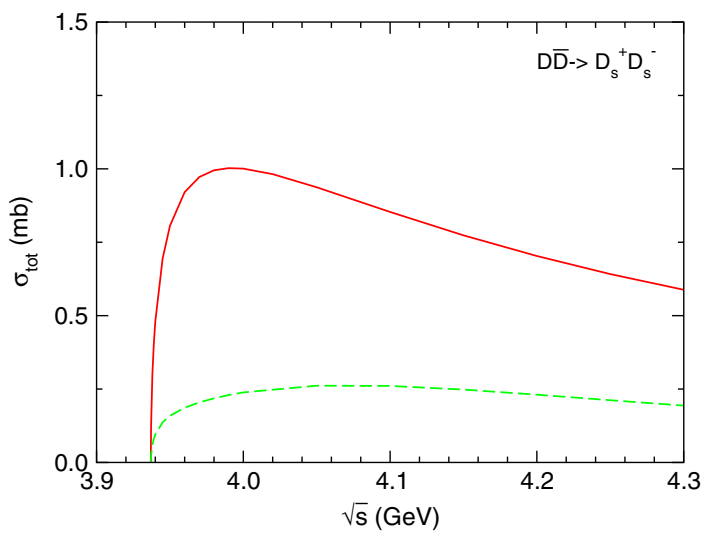

FIG. 13 (color online). Cross section for $D \bar{D} \rightarrow D_{s}^{+} D_{s}^{-}$as a function of $\sqrt{s}$ calculated from the meson-exchange model (solid line) and the quark model (dashed line).

\section{SUMMARY}

We have presented predictions for the reactions $\bar{p} p \rightarrow$ $D \bar{D}$ and $\bar{p} p \rightarrow D_{s}^{+} D_{s}^{-}$based on a model calculation performed within the baryon-exchange picture in close analogy to the Jülich analysis of the reaction $\bar{p} p \rightarrow \bar{K} K$ $[15,16]$, connecting those processes via $S U(4)$ symmetry.

Effects of the interaction in the inital $\bar{p} p$ channel which play a crucial role for quantitative predictions were taken into account. Furthermore, the Jülich $\pi \pi-\bar{K} K$ model [37] was extended to higher energies by including also the $D \bar{D}$ and $D_{s}^{+} D_{s}^{-}$channels so that even effects from the final-state interaction could be investigated. In particular, the coupling between the $D \bar{D}$ and $D_{s}^{+} D_{s}^{-}$systems, facilitated by the FSI, allowed us to obtain predictions for $\bar{p} p \rightarrow D_{s}^{+} D_{s}^{-}$, i.e., for the production of charmed strange mesons in $\bar{N} N$ collisions, which is only possible via a two-step process.

The cross sections for $\bar{p} p \rightarrow D \bar{D}$ were found to be on the order of $10^{-2}-10^{-1} \mu b$, and they turned out to be comparable to those predicted by other model calculations in the literature. The cross section for a $D_{s}^{+} D_{s}^{-}$pair was found to be roughly of the same order of magnitude, despite of the fact that its production in $\bar{p} p$ scattering requires a two-step process.

As before in our study of the reaction $\bar{p} p \rightarrow \bar{\Lambda}_{c}^{-} \Lambda_{c}^{+}$[20], we investigated an alternative mechanism for the charm production. This was done in the form of a $\bar{p} p \rightarrow D \bar{D}$ transition potential derived in a constituent quark model where two (up or down) quark pairs are annihilated and a charmed quark pair is created. It turned out that the $\bar{p} p \rightarrow$ $D \bar{D}$ cross sections predicted by the mechanism based on the quark picture are essentially of the same order of magnitude as those that we obtained from baryon exchange.

Our results suggest that the reactions $\bar{p} p \rightarrow D \bar{D}$ and $\bar{p} p \rightarrow D_{s}^{+} D_{s}^{-}$take place predominantly in the $s$ wave, at least for excess energies below $100 \mathrm{MeV}$, say. But we should mention that there is a well-established $p$-wave resonance, the $\psi(3770)\left(J^{P C}=1^{--}\right)$, which is seen as a pronounced structure in $e^{+} e^{-} \rightarrow D \bar{D}$ [61,62], for example, and which decays almost exclusively (i.e., to $93_{-9}^{+8} \%$ ) into $D \bar{D}$ [63]. This resonance is located at only around $35 \mathrm{MeV}$ above the $D \bar{D}$ threshold. We did not include it in the present study because at the moment the strength of the coupling of the $\psi(3770)$ to $\bar{p} p$ is not that well known [64]. But its impact should be definitely explored in any more refined studies of $\bar{p} p \rightarrow D \bar{D}$ in the future. Evidently, it would be also interesting to examine the energy range in question in pertinent experiments, which could be performed at FAIR, in order to see whether there is a signal of this resonance.

\section{ACKNOWLEDGMENTS}

The work of G. K. was partially financed by CNPq and FAPESP (Brazilian agencies). The work was also partially 
supported by the EU Integrated Infrastructure Initiative HadronPhysics3 (Contract No. 283286).

\section{APPENDIX A: INTERACTION LAGRANGIANS}

Here we list the specific interaction Lagrangians, which are used to derive the interactions. The baryon-baryonmeson couplings that enter the $\bar{N} N \rightarrow M_{1} M_{2}$ transition potentials are given by

$\mathcal{L}_{B^{\prime} B P}=\frac{f_{B^{\prime} B P}}{m_{P}} \bar{\Psi}_{B^{\prime}}(x) \gamma^{5} \gamma^{\nu} \Psi_{B}(x) \partial_{\nu} \Phi_{P}(x)+$ H.c.,

$\mathcal{L}_{B^{\prime} \tilde{B} P}=\frac{f_{B^{\prime} \tilde{B} P}}{m_{P}} \bar{\Psi}_{B^{\prime}}(x) \Psi_{\tilde{B}}^{\mu}(x) \partial_{\mu} \Phi_{P}(x)+$ H.c.

In Eq. (A1) $\Psi_{B}\left(B=N, \Lambda, \Sigma, \Lambda_{c}, \Sigma_{c}\right)$ are the octet (spin-1/2) baryon field operators and $\Psi_{\tilde{B}}^{\mu} \quad\left[\tilde{B}=\Delta, \Sigma^{*}(1385)\right.$, $\left.\Sigma_{c}^{*}(2520)\right]$ the decuplet (spin-3/2) field operators, while $\Phi_{P}$ is the field operator for pseudoscalar mesons. Explicit expression for the resulting transition potentials can be found in Appendix A of Ref. [14].

The employed three-meson couplings for the various $M_{1} M_{2} \rightarrow M_{3} M_{4}$ potentials and transitions are

$$
\begin{aligned}
\mathcal{L}_{P P S} & =\frac{g_{P P S}}{m_{P}} \partial^{\mu} \Phi_{P}(x) \partial_{\mu} \Phi_{P}(x) \Phi_{S}(x), \\
\mathcal{L}_{P P V} & =g_{P P V} \Phi_{P}(x) \partial_{\mu} \Phi_{P}(x) \Phi_{V}^{\mu}(x), \\
\mathcal{L}_{P P T} & =g_{P P T} \partial_{\mu} \Phi_{P}(x) \partial_{\nu} \Phi_{P}(x) \Phi_{T}^{\mu \nu}(x),
\end{aligned}
$$

for the coupling of a scalar $(S)$, vector $(V)$, or tensor $(T)$ meson to pseudoscalar mesons. Expressions for the transition potentials in the meson-meson sector can be found in the Appendix of Ref. [37]. Note that in the equations above only the space-spin part is given. There is also an isospin dependence that has to be taken into account in the actual calculation. The SU(4) flavor structure leads to the characteristic relations between the coupling constants. For the vertices involving baryons, they are given by

$$
\begin{aligned}
& f_{\Lambda_{c}^{+} N D}=f_{\Lambda N K}=-\frac{1}{\sqrt{3}}(1+2 \alpha) f_{N N \pi}, \\
& f_{\Sigma_{c} N D}=f_{\Sigma N K}=(1-2 \alpha) f_{N N \pi}, \\
& f_{N \Sigma_{c}^{*} D}=f_{N \Sigma^{*} K}=-\frac{1}{\sqrt{6}} f_{N \Delta \pi},
\end{aligned}
$$

with $\alpha$ the $F /(F+D)$ ratio. The coupling constants for the meson-meson interaction are discussed in detail below.

\section{APPENDIX B: QUARK MODEL EXPRESSIONS}

The result for the $\bar{p} p \rightarrow D \bar{D}$ transition potential presented in Eq. (7) is obtained from the matrix element $\left\langle\Psi_{f}\left|V_{A}\right| \Psi_{i}\right\rangle$ provided in Eq. (A2) of Ref. [28], with the relative $\bar{p} p$ wave function $\varphi_{E}(\mathbf{r})$ given by a plane wave with momentum $\mathbf{p}$ and after summing over spin-isospin indices. The explicit expressions of the form factors $h_{1}(t)=h_{1}\left(\mathbf{p}, \mathbf{p}^{\prime}\right)$ and $h_{2}(t)=h_{2}\left(\mathbf{p}, \mathbf{p}^{\prime}\right)$ that appear in Eq. (7) are given by

$$
\begin{gathered}
h_{1}\left(\mathbf{p}, \mathbf{p}^{\prime}\right)=-\frac{b_{N}^{2}}{3 b_{N}^{2}+2 b_{M}^{2}} h\left(\mathbf{p}, \mathbf{p}^{\prime}\right), \\
h_{2}\left(\mathbf{p}, \mathbf{p}^{\prime}\right)=\left[1-2 \beta \frac{b_{M}^{2}}{\left(3 b_{N}^{2}+2 b_{M}^{2}\right)}\right] h\left(\mathbf{p}, \mathbf{p}^{\prime}\right),
\end{gathered}
$$

with $\beta=m_{q} /\left(m_{q}+m_{h}\right), m_{q}=\left(m_{u}, m_{d}\right), m_{h}=\left(m_{c}, m_{s}\right)$, and

$$
\begin{aligned}
h\left(\mathbf{p}, \mathbf{p}^{\prime}\right)= & 24 \lambda C_{A} \frac{4 \pi}{Q}\left(\frac{\alpha_{A}}{m_{G}^{2}}\right)^{2} v\left(\mathbf{p}, \mathbf{p}^{\prime}\right) \\
& \times 4 \pi \int_{0}^{\infty} d z z^{3} j_{1}(Q z) f(z),
\end{aligned}
$$

where $C_{A}=4 / 27$ comes from summing over color indices, $\lambda=1$ for $\bar{p} p \rightarrow \bar{D}^{0} D^{0}\left(K^{+} K^{-}\right), \quad \lambda=-1$ for $\bar{p} p \rightarrow \bar{D}^{+} D^{-}\left(\bar{K}^{0} K^{0}\right)$,

$$
\mathbf{Q}=\left[1-2 \beta \frac{b_{M}^{2}}{\left(3 b_{N}^{2}+2 b_{M}^{2}\right)}\right] \mathbf{p}^{\prime}-\frac{b_{N}^{2}}{3 b_{N}^{2}+2 b_{M}^{2}} \mathbf{p},
$$

and

$$
\begin{aligned}
v\left(\mathbf{p}, \mathbf{p}^{\prime}\right)= & {\left[\frac{12 b_{M}^{2}}{\left(3 b_{N}^{2}+5 b_{M}^{2}\right)\left(3 b_{N}^{2}+2 b_{M}^{2}\right)}\right]^{3 / 2} } \\
& \times \exp \left[-\frac{b_{N}^{2} b_{M}^{2}}{9 b_{N}^{2}+6 b_{M}^{2}}\left(\mathbf{p}-3 \beta \mathbf{p}^{\prime}\right)^{2}\right], \\
f(z)= & \frac{1}{z^{3}}(1+\mu z) \\
& \times \exp \left[-\mu z-\left(\frac{2 b_{N}^{2}+b_{M}^{2}}{3 b_{N}^{2}+2 b_{M}^{2}}\right) z^{2}\right] .
\end{aligned}
$$

Here, $\mu=m_{h}$ comes from the (static) heavy quark propagator-see Fig. 4-and $b_{N}$ and $b_{M}$ are the Gaussian widths of the nucleon and meson wave functions, related to their rms radii by $b_{N}=\left\langle r_{N}^{2}\right\rangle^{1 / 2}$ and $b_{M}=\sqrt{8 / 3}\left\langle r_{M}^{2}\right\rangle^{1 / 2}$. Also, in Eq. (B3) the factor $\left(\alpha_{A} / m_{G}^{2}\right)$ indicates the strengths of light-quark pair annihilation and heavy-quark pair creation.

For the $\bar{K} K \rightarrow D_{s}^{+} D_{s}^{-}$transition potential, the explicit expression is

$$
\begin{aligned}
V_{Q}^{\bar{K} K \rightarrow D_{s}^{+} D_{s}^{-}}\left(\mathbf{p}, \mathbf{p}^{\prime}\right)= & 4 \pi \frac{\alpha_{A}}{m_{G}^{2}} C_{K D_{s}}\left(\frac{2 b_{K} b_{D_{s}}}{b_{K}^{2}+b_{D_{s}}^{2}}\right)^{3} \\
& \times \exp \left[-\frac{1}{4} \frac{b_{K}^{2} b_{D_{s}}^{2}}{b_{K}^{2}+b_{D_{s}}^{2}}\left(m \mathbf{p}-M \mathbf{p}^{\prime}\right)^{2}\right],
\end{aligned}
$$


where $\mathbf{p}$ and $\mathbf{p}^{\prime}$ are the initial and final state c.m. momenta, $C_{K D_{S}}=4 / 9$ comes from color,

$$
m=\frac{2 m_{s}}{m_{s}+m_{q}}, \quad M=\frac{2 m_{s}}{m_{c}+m_{s}},
$$

and the $b$ 's are Gaussian size parameters, related to the meson rms radii as above. For the reaction $D \bar{D} \rightarrow D_{s}^{+} D_{s}^{-}$, the color factor is the same, $b_{K} \rightarrow b_{D}$, and the masses $m$ and $M$ are replaced by

$$
m=\frac{2 m_{c}}{m_{c}+m_{q}}, \quad M=\frac{2 m_{c}}{m_{c}+m_{s}} .
$$

The values of parameters we use are standard, namely, the constituent quark masses are taken to be $m_{u}=$ $m_{d}=330 \mathrm{MeV}, m_{s}=550 \mathrm{MeV}, m_{c}=1600 \mathrm{MeV}$, and the meson Gaussian size parameters are such that $\left\langle r_{M}^{2}\right\rangle^{1 / 2}=0.4 \mathrm{fm}$ and $\left\langle r_{N}^{2}\right\rangle^{1 / 2}=0.55 \mathrm{fm}$. Regarding the effective coupling strength, we use the value $\alpha_{A} / m_{G}^{2}=$ $0.12 \mathrm{fm}^{2}$, which we fixed from a fit to the $\bar{p} p \rightarrow K^{-} K^{+}$ cross section, i.e., in a reaction where a pair of strange quarks is produced. This is very close to the original value of Kohno and Weise [28], who adopted the value $\alpha_{A} / m_{G}^{2}=$ $0.15 \mathrm{fm}^{2}$ in their calculation of the same reaction. As already noted in our study of the reaction $\bar{p} p \rightarrow \bar{\Lambda}_{c}^{-} \Lambda_{c}^{+}$ [20], this effective coupling depends implicitly on the effective gluon propagator, i.e., on the square of the energy transfer from the initial to final quark pair. Heuristically, this energy transfer corresponds roughly to the masses of the produced constituent quarks, i.e., $m_{G} \approx 2 m_{q}$. Thus, we assume the effective coupling strength for charm production to be reduced by the ratio of the constituent quark masses of the strange and the charmed quark squared, $\left(m_{s} / m_{c}\right)^{2} \approx(550 / 1600)^{2} \approx 1 / 9$ as compared to the one used for strangeness production.

\section{Appendix C: SU(4) RELATIONS FOR THE MESON-MESON INTERACTION}

The general form of the $\mathrm{SU}(4)$ invariant Lagrangian is

$$
\begin{aligned}
\mathcal{L}_{M M M}= & g_{\{15\}}\left[-\alpha \operatorname{Tr}\left(\left[M_{\{15\}}, M_{\{15\}}\right] M_{\{15\}}\right)\right. \\
& \left.+(1-\alpha) \operatorname{Tr}\left(\left\{M_{\{15\}}, M_{\{15\}}\right\} M_{\{15\}}\right)\right] \\
& +g_{\{15\}\{15\}\{1\}}(1-\alpha) \operatorname{Tr}\left(\left\{M_{\{15\}}, M_{\{15\}}\right\} M_{\{1\}}\right) \\
& +g_{\{15\}\{1\}\{15\}}(1-\alpha) \operatorname{Tr}\left(\left\{M_{\{15\}}, M_{\{1\}}\right\} M_{\{15\}}\right) \\
& +g_{\{1\}}(1-\alpha) \operatorname{Tr}\left(\left\{M_{\{1\}}, M_{\{1\}}\right\} M_{\{1\}}\right),
\end{aligned}
$$

where $M_{\{15\}}\left(M_{\{1\}}\right)$ stands for the SU(4) meson-15-plet (-singlet) matrix. For pseudo-scalar $(P)$ and vector $(V)$ mesons, $M_{\{15\}}$ is a $4 \times 4$ matrix of the form

$$
\begin{aligned}
& P=\left(\begin{array}{cccc}
\frac{\pi^{0}}{\sqrt{2}}+\frac{\eta}{\sqrt{6}}+\frac{\eta_{c}}{\sqrt{12}} & \pi^{+} & K^{+} & \bar{D}^{0} \\
\pi^{-} & -\frac{\pi^{0}}{\sqrt{2}}+\frac{\eta}{\sqrt{6}}+\frac{\eta_{c}}{\sqrt{12}} & K^{0} & D^{-} \\
K^{-} & \bar{K}^{0} & -\sqrt{\frac{2}{3} \eta+\frac{\eta_{c}}{\sqrt{12}}} & D_{s}^{-} \\
D^{0} & D^{+} & D_{s}^{+} & -\frac{3 \eta_{c}}{\sqrt{12}}
\end{array}\right), \\
& V=\left(\begin{array}{cccc}
\frac{\rho^{0}}{\sqrt{2}}+\frac{\omega_{8}}{\sqrt{6}+\frac{\omega_{15}}{\sqrt{12}}} & \rho^{+} & K^{*+} & \bar{D}^{* 0} \\
\rho^{-} & -\frac{\rho^{0}}{\sqrt{2}}+\frac{\omega_{8}}{\sqrt{6}}+\frac{\omega_{15}}{\sqrt{12}} & K^{* 0} & D^{*-} \\
K^{*-} & \bar{K}^{* 0} & -\sqrt{\frac{2}{3}} \omega_{8}+\frac{\omega_{15}}{\sqrt{12}} & D_{s}^{*-} \\
D^{* 0} & D^{*+} & D_{s}^{*+} & -\frac{3 \omega_{15}}{\sqrt{12}}
\end{array}\right) .
\end{aligned}
$$

For the construction of the $D \bar{D}$ and $D_{s}^{+} D_{s}^{-}$interactions and the transition potentials to the $\pi \pi, \pi \eta$, and $\bar{K} K$ channels, we need three-meson vertices involving charmed mesons of the kind $P P V$. The $P P V$ vertices involve only $F$ type coupling $(\alpha=1)$ if we require charge conjugation invariance, and, therefore, in this case there is no singlet coupling; cf. Eq. (C1).

Based on the assumed SU(4) symmetry, all relevant three-meson coupling constants can be derived from the empirically known $\pi \pi \rho$ coupling. In the Jülich model [37], the value $g_{\pi \pi \rho}=6.04$ is used. The coupling constants of the other vertices that follow from this value are listed in Table II.

Let us make some more comments about the coupling constants at the three-meson vertices, specifically with regard to the imposed ideal mixing between the octet and singlet. $S U(4)$ symmetry implies the following for the vector meson coupling constants relevant for our study: 
TABLE II. Vertex parameters for $t$-channel exchanges. Relations between coupling constants are obtained using SU(4) and ideal mixing between the octet and singlet.

\begin{tabular}{lcc}
\hline \hline Vertex & $g$ & $\Lambda[\mathrm{MeV}]$ \\
\hline$\pi \pi \rho$ & 6.04 & 1355 \\
$\pi K K^{*}$ & $g_{\pi K K^{*}}=g_{\pi \bar{K} \overline{K^{*}}}=-\frac{1}{2} g_{\pi \pi \rho}$ & 1900 \\
$K K \rho$ & $g_{K K \rho}=g_{\bar{K} \bar{K} \rho}=\frac{1}{2} g_{\pi \pi \rho}$ & 1850 \\
$K K \omega$ & $g_{K K \omega}=-g_{\bar{K} \bar{K} \omega}=\frac{1}{2} g_{\pi \pi \rho}$ & 2800 \\
$K K \phi$ & $g_{K K \phi}=-g_{\bar{K} \bar{K} \phi}=\frac{1}{\sqrt{2}} g_{\pi \pi \rho}$ & 2800 \\
$\eta K K^{*}$ & $g_{\eta K K^{*}}=-g_{\eta \bar{K}} \overline{K^{*}}=-\frac{\sqrt{3}}{2} g_{\pi \pi \rho}$ & 3290 \\
$\pi D D^{*}$ & $g_{\pi \bar{D}} \overline{D^{*}}=g_{\pi D D^{*}}=-\frac{1}{2} g_{\pi \pi \rho}$ & 3100 \\
$D K D_{s}^{*}$ & $g_{\bar{D} K D_{s}^{*-}}=-g_{D \bar{K} D_{s}^{*+}}=-\frac{1}{\sqrt{2}} g_{\pi \pi \rho}$ & 3100 \\
$D D \rho$ & $g_{\bar{D} \bar{D} \rho}=g_{D D \rho}=\frac{1}{2} g_{\pi \pi \rho}$ & 1850 \\
$D D \omega$ & $g_{\bar{D} \bar{D} \omega}=-g_{D D \omega}=\frac{1}{2} g_{\pi \pi \rho}$ & 2800 \\
$D D \psi$ & $g_{\bar{D} \bar{D} \psi}=-g_{D D \psi}=-\frac{1}{\sqrt{2}} g_{\pi \pi \rho}$ & 4000 \\
$D_{s} K D^{*}$ & $g_{D_{s}^{+} K D^{*}}=-g_{D_{s}^{-} \bar{K} \bar{D}^{*}}=\frac{1}{\sqrt{2}} g_{\pi \pi \rho}$ & 3100 \\
$D D_{s} K^{*}$ & $g_{\bar{D} D_{s}^{-} K^{*}}=-g_{D D_{s}^{+} K^{*}}=\frac{1}{\sqrt{2}} g_{\pi \pi \rho}$ & 1900 \\
$D_{s} D_{s} \phi$ & $g_{D_{s}^{-} D_{s}^{-} \phi}=-g_{D_{s}^{+} D_{s}^{+} \phi}=-\frac{1}{\sqrt{2}} g_{\pi \pi \rho}$ & 2800 \\
$D_{s} D_{s} \psi$ & $g_{D_{s}^{-} D_{s}^{-} \psi}=-g_{D_{s}^{+} D_{s}^{+} \psi}=-\frac{1}{\sqrt{2}} g_{\pi \pi \rho}$ & 4000 \\
\hline \hline
\end{tabular}

$$
\begin{array}{rlrl}
g_{K K \omega_{8}} & =\sqrt{3} g_{K K \rho}=\frac{\sqrt{3}}{2} g_{\pi \pi \rho}, \quad g_{K K \omega_{15}}=0, \\
g_{\bar{D} \bar{D} \omega_{8}} & =\sqrt{\frac{1}{3}} g_{K K \rho}, & g_{\bar{D} \bar{D} \omega_{15}} & =\sqrt{\frac{8}{3}} g_{K K \rho}, \\
g_{D_{s}^{-} D_{s}^{-} \omega_{8}} & =-\frac{2}{\sqrt{3}} g_{K K \rho}, & & g_{D_{s}^{-} D_{s}^{-} \omega_{15}}=\sqrt{\frac{8}{3}} g_{K K \rho}, \\
g_{D_{s}^{+} D_{s}^{+} \omega_{8}} & =\frac{2}{\sqrt{3}} g_{K K \rho}, & & g_{D_{s}^{+} D_{s}^{+} \omega_{15}}=-\sqrt{\frac{8}{3}} g_{K K \rho} .
\end{array}
$$

Assuming ideal mixing of the $\omega_{15}, \omega_{8}$, and $\omega_{1}$ one obtains for the coupling constants of the physical $\omega, \phi$, and $J / \psi$

$$
\begin{aligned}
& g_{\bar{D} \bar{D} \omega}=\sqrt{\frac{1}{2}} g_{\bar{D} \bar{D} \omega_{1}}+\sqrt{\frac{1}{3}} g_{\bar{D} \bar{D} \omega_{8}}+\sqrt{\frac{1}{6}} g_{\bar{D} \bar{D} \omega_{15}}, \\
& g_{\bar{D} \bar{D} \phi}=-\sqrt{\frac{1}{4}} g_{\bar{D} \bar{D} \omega_{1}}+\sqrt{\frac{2}{3}} g_{\bar{D} \bar{D} \omega_{8}}-\sqrt{\frac{1}{12}} g_{\bar{D} \bar{D} \omega_{15}}, \\
& g_{\bar{D} \bar{D} \psi}=\sqrt{\frac{1}{4}} g_{\bar{D} \bar{D} \omega_{1}}-\sqrt{\frac{3}{4}} g_{\bar{D} \bar{D} \omega_{15}} .
\end{aligned}
$$

The same relation holds also for the $K$ meson and for the $D_{s}^{-}$and $D_{s}^{+}$. In case of the $K$ meson, the coupling constant $g_{K K \omega}$ is given by that of $g_{K K \omega_{8}}$ alone, since there is no singlet coupling for PPV vertices as mentioned above:

$$
g_{K K \omega}=\sqrt{\frac{1}{3}} g_{K K \omega_{8}}=g_{K K \rho} .
$$

In case of the $D$ meson, the coupling constant is given by

$$
\begin{aligned}
& g_{\bar{D} \bar{D} \omega}=\sqrt{\frac{1}{3}} g_{\bar{D} \bar{D} \omega_{8}}+\sqrt{\frac{1}{6}} g_{\bar{D} \bar{D} \omega_{15}}=g_{K K \rho} \\
& g_{\bar{D} \bar{D} \psi}=-\sqrt{\frac{3}{4}} g_{\bar{D} \bar{D} \omega_{15}}=-\sqrt{2} g_{K K \rho},
\end{aligned}
$$

and for the $D_{s}$ meson,

$$
\begin{aligned}
g_{D_{s}^{-} D_{s}^{-} \phi} & =\sqrt{\frac{2}{3}} g_{D_{s}^{-} D_{s}^{-} \omega_{8}}-\sqrt{\frac{1}{12}} g_{D_{s}^{-} D_{s}^{-} \omega_{15}} \\
& =-\sqrt{2} g_{K K \rho} \\
g_{D_{s}^{-} D_{s}^{-} \psi} & =-\sqrt{\frac{3}{4}} g_{D_{s}^{-} D_{s}^{-} \omega_{15}}=-\sqrt{2} g_{K K \rho} .
\end{aligned}
$$

The Jülich $\pi \pi-\bar{K} K$ potential contains also vertex form factors $F$ that are meant to take into account the extended hadron structure and are parametrized in the conventional monopole or dipole form $[36,37]$. In the present extension to the $D \bar{D}$ and $D_{s}^{+} D_{s}^{-}$systems, the cutoff masses appearing in those form factors for the various three-meson vertices are mostly taken over from Ref. [37]. In particular, for vertices involving vector mesons without charm $\left(\rho, \omega, \phi, K^{*}\right)$, we make the assumption that $F_{D D V}\left(\boldsymbol{q}_{V}^{2}\right) \approx F_{K K V}\left(\boldsymbol{q}_{V}^{2}\right)$ and/or $F_{D_{s} D_{s} V}\left(\boldsymbol{q}_{V}^{2}\right) \approx F_{K K V}\left(\boldsymbol{q}_{V}^{2}\right)$; i.e., we use the same cutoff masses for the same exchange particles-a prescription that is guided by the notion that those form factors parametrize predominantly the off-mass-shell behavior of the exchanged particles. For the additional vertices that concern the exchange of a $D^{*}(2009)$ or $D_{s}^{*}(2112)$ or of a $\psi(3096)$, we adopt cutoff masses that are about $1 \mathrm{GeV}$ larger than the mass of the exchange particle. A compilation of the cutoff masses employed at the various three-meson vertices is provided in Table II.

In our model calculation, we use $P P V$ coupling constants that are determined fully by SU(4) symmetry. In our opinion, the difference of those values to the ones deduced from available experimental information is not very large and, thus, does not really warrant a departure from SU(4) at present. But let us review the situation briefly here. The $D D \rho$ coupling constant was determined in Refs. $[65,66]$ based on the vector dominance model and found to be $g_{D D \rho}=2.52-2.8$. This value, which was subsequently adopted in several investigations [67-69], is only marginally smaller than the one which follows from assuming $\mathrm{SU}(4)$ symmetry. The same is true for the $D D \omega$ coupling constant, found to be $g_{D D \omega}=-2.84$ in Ref. [66], likewise derived within the vector dominance model. In Ref. [69] the value $g_{\pi D D^{*}}=5.56$ is cited, derived from the measured decay width of the $D^{*}$ meson. Here the corresponding SU(4) coupling constant is roughly a factor 2 smaller. Deviations from the SU(4) symmetry are also discussed in Refs. [57,70-72]. 
[1] W. Erni et al. (Panda Collaboration), arXiv:0903.3905.

[2] B. Friman, C. Höhne, J. Knoll, S. Leupold, J. Randrup, R. Rapp, and P. Senger, Lect. Notes Phys. 814, 849 (2011).

[3] U. Wiedner, Prog. Part. Nucl. Phys. 66, 477 (2011).

[4] T. Matsui and H. Satz, Phys. Lett. B 178, 416 (1986).

[5] S. J. Brodsky, I. A. Schmidt, and G. F. de Teramond, Phys. Rev. Lett. 64, 1011 (1990).

[6] S. H. Lee and C. M. Ko, Phys. Rev. C 67, 038202 (2003).

[7] G. Krein, A. W. Thomas, and K. Tsushima, Phys. Lett. B 697, 136 (2011).

[8] K. Tsushima, D. H. Lu, G. Krein, and A. W. Thomas, Phys. Rev. C 83, 065208 (2011).

[9] G. Krein, J. Phys. Conf. Ser. 422, 012012 (2013).

[10] C. B. Dover and S. H. Kahana, Phys. Rev. Lett. 39, 1506 (1977).

[11] K. Tsushima, D. H. Lu, A. W. Thomas, K. Saito, and R. H. Landau, Phys. Rev. C 59, 2824 (1999).

[12] C. Garcia-Recio, J. Nieves, and L. Tolos, Phys. Lett. B 690, 369 (2010).

[13] C. Garcia-Recio, J. Nieves, L. L. Salcedo, and L. Tolos, Phys. Rev. C 85, 025203 (2012).

[14] T. Hippchen, J. Haidenbauer, K. Holinde, and V. Mull, Phys. Rev. C 44, 1323 (1991).

[15] V. Mull, J. Haidenbauer, T. Hippchen, and K. Holinde, Phys. Rev. C 44, 1337 (1991).

[16] V. Mull and K. Holinde, Phys. Rev. C 51, 2360 (1995)

[17] J. Haidenbauer, G. Krein, U.-G. Meißner, and A. Sibirtsev, Eur. Phys. J. A 33, 107 (2007).

[18] J. Haidenbauer, G. Krein, U.-G. Meißner, and A. Sibirtsev, Eur. Phys. J. A 37, 55 (2008).

[19] J. Haidenbauer, G. Krein, U.-G. Meißner, and L. Tolos, Eur. Phys. J. A 47, 18 (2011).

[20] J. Haidenbauer and G. Krein, Phys. Lett. B 687, 314 (2010).

[21] D. Hadjimichef, J. Haidenbauer, and G. Krein, Phys. Rev. C 63, 035204 (2001).

[22] D. Hadjimichef, J. Haidenbauer, and G. Krein, Phys. Rev. C 66, 055214 (2002).

[23] J. Haidenbauer, T. Hippchen, K. Holinde, B. Holzenkamp, V. Mull, and J. Speth, Phys. Rev. C 45, 931 (1992); Phys. Rev. C 46 (1992) 2158.

[24] V. Mull, K. Holinde, and J. Speth, Phys. Lett. B 275, 12 (1992).

[25] J. Haidenbauer, K. Holinde, V. Mull, and J. Speth, Phys. Rev. C 46, 2158 (1992).

[26] M. Kohno and W. Weise, Phys. Lett. B 179, 15 (1986).

[27] M. A. Alberg, E. M. Henley, L. Wilets, and P. D. Kunz, Nucl. Phys. A560, 365 (1993).

[28] M. Kohno and W. Weise, Nucl. Phys. A454, 429 (1986).

[29] P. Kroll, B. Quadder, and W. Schweiger, Nucl. Phys. B316, 373 (1989).

[30] A. B. Kaidalov and P. E. Volkovitsky, Z. Phys. C 63, 517 (1994).

[31] B. Kerbikov and D. Kharzeev, Phys. Rev. D 51, 6103 (1995).

[32] A. I. Titov and B. Kämpfer, Phys. Rev. C 78, 025201 (2008).

[33] A. Khodjamirian, C. Klein, T. Mannel, and Y. M. Wang, Eur. Phys. J. A 48, 31 (2012).

[34] A. T. Goritschnig, B. Pire, and W. Schweiger, Phys. Rev. D 87, 014017 (2013); Phys. Rev. D88, 079903(E) (2013).
[35] J. Haidenbauer and G. Krein, Few-Body Syst. 50, 183 (2011).

[36] D. Lohse, J. W. Durso, K. Holinde, and J. Speth, Nucl. Phys. A516, 513 (1990).

[37] G. Janssen, B. C. Pearce, K. Holinde, and J. Speth, Phys. Rev. D 52, 2690 (1995).

[38] J. Haidenbauer, K. Holinde, and M. B. Johnson, Phys. Rev. C 45, 2055 (1992).

[39] S. P. Denisov, S. V. Donskov, Yu. P. Gorin, A. I. Petrukhin, Yu. D. Prokoshkin, and D. A. Stoyanova, Nucl. Phys. B65, 1 (1973).

[40] K. J. Foley, S. Lindenbaum, W. Love, S. Ozaki, J. Russell, and L. Yuan, Phys. Rev. Lett. 11, 503 (1963).

[41] P. Astbury et al., Phys. Lett. 23, 160 (1966).

[42] A. Berglund et al., Nucl. Phys. B176, 346 (1980).

[43] G. D. Patel et al., Z. Phys. C 12, 189 (1982).

[44] P. S. Gregory, P. Johnson, P. Mason, H. Muirhead, G. Warren, G. Ekspong, S. O. Holmgren, S. Nilsson, R. Stenbacka, and Ch. Walck, Nucl. Phys. B119, 60 (1977).

[45] P. Johnson et al., Nucl. Phys. B173, 77 (1980).

[46] I. Ambats, D. Ayres, R. Diebold, A. Greene, S. Kramer, A. Lesnik, D. Rust, C. Ward, A. Wicklund, and D. Yovanovitch, Phys. Rev. D 9, 1179 (1974).

[47] J. S. Russ et al., Phys. Rev. D 15, 3139 (1977).

[48] J. G. Lee et al., Nucl. Phys. B52, 292 (1973).

[49] T. Buran et al., Nucl. Phys. B97, 11 (1975).

[50] B. Holzenkamp, K. Holinde, and J. Speth, Nucl. Phys. A500, 485 (1989).

[51] J. Haidenbauer, K. Holinde, and A. W. Thomas, Phys. Rev. C 45, 952 (1992).

[52] M. A. Mandelkern, R. R. Burns, P.E. Condon, and J. Schultz, Phys. Rev. D 4, 2658 (1971).

[53] F. Sai, S. Sakamoto, and S. S. Yamamoto, Nucl. Phys. B213, 371 (1983).

[54] T. Tanimori, T. Fujii, T. Kageyama, K. Nakamura, F. Sai, S. Sakamoto, S. Sato, T. Takahashi, S. Yamamoto, and Y. Takada, Phys. Rev. Lett. 55, 1835 (1985).

[55] Y. Sugimoto et al., Phys. Rev. D 37, 583 (1988).

[56] A. Hasan et al., Nucl. Phys. B378, 3 (1992).

[57] G. Krein, Proc. Sci., ConfinementX (2012) 144.

[58] Y.-R. Liu, M. Oka, M. Takizawa, X. Liu, W.-Z. Deng, and S. -L. Zhu, Phys. Rev. D 82, 014011 (2010).

[59] J. Haidenbauer, K. Holinde, and J. Speth, Nucl. Phys. A562, 317 (1993).

[60] P. D. Barnes et al. (PS185 Collaboration), Phys. Lett. B 402, 227 (1997).

[61] M. Ablikim et al., Phys. Lett. B 668, 263 (2008).

[62] V. V. Anashin et al., Phys. Lett. B 711, 292 (2012).

[63] J. Beringer et al. (Particle Data Group), Phys. Rev. D 86, 010001 (2012).

[64] M. Ablikim et al. (BESIII Collaboration), arXiv:1403.6011.

[65] S. G. Matinyan and B. Müller, Phys. Rev. C 58, 2994 (1998); note that the actual coupling constant given in this reference is a factor of 2 larger due to the difference of the definitions.

[66] Z. Lin and C. M. Ko, Phys. Rev. C 62, 034903 (2000).

[67] Z. Lin, C. M. Ko, and B. Zhang, Phys. Rev. C 61, 024904 (2000).

[68] Z. Lin, T. G. Di, and C. M. Ko, Nucl. Phys. A689, 965 (2001). 
[69] W. Liu and C.M. Ko, Phys. Lett. B 533, 259 (2002).

[70] B. El-Bennich, G. Krein, L. Chang, C. D. Roberts, and D. J. Wilson, Phys. Rev. D 85, 031502 (2012).
[71] M. E. Bracco, M. Chiapparini, F. S. Navarra, and M. Nielsen, Prog. Part. Nucl. Phys. 67, 1019 (2012).

[72] K. U. Can, G. Erkol, M. Oka, and T. Takahashi, Phys. Lett. B 719, 103 (2013). 